\title{
Bringing Structural Implications and Deep Learning Based Drug Identification for KRAS Mutants
}

\author{
Aamir Mehmood ${ }^{1,3}$ | Aman Chandra Kaushik ${ }^{2}$ Qiankun Wang ${ }^{1}$ Cheng-Dong Li ${ }^{1}$ | Dong- \\ Qing Wei ${ }^{1,3 *}$
}

${ }^{1}$ State Key Laboratory of Microbial Metabolism and School of Life Sciences and Biotechnology, Shanghai Jiao Tong University, 800 Dongchuan Road, Shanghai 200240, China

${ }^{2}$ Wuxi School of Medicine, Jiangnan University, Li Lake Avenue, Wuxi 214122, Jiangsu, China

${ }^{3}$ Peng Cheng Laboratory, Vanke Cloud City Phase I Building 8, Xili Street, Nanshan District, Shenzhen, Guangdong, 518055, P.R China

\section{*Correspondence}

Dong-Qing Wei, Ph.D., Professor, Department of Bioinformatics and Biostatistics College of Life Sciences and Biotechnology, The State Key Laboratory of Microbial Metabolism, Shanghai Jiao Tong University. dqwei@sjtu.edu.cn

800 Dongchuan Road Shanghai, Minhang District China, 200240 Tel: 86-21-3420-4573

\section{SUPPLEMEATRY DATA}

\section{Chapter 1}

Table S1. Anticancer drugs list from Cancer Cell Line Encyclopedia (CCLE) among the KRAS mutated and non-KRAS mutations in colon cancer cell lines.

\begin{tabular}{|l|l|l|l|l|l|l|}
\hline Drug & Drug Target & Effect size & P-value & FDR\% & No. of altered cell lines & Tissue analysis \\
\hline PLX-4720 & BRAF & 1.07 & 0.00096 & 3.99 & 25 & COREAD \\
\hline Vorinostat & HDAC inhibitor Class I, IIa, IIb, IV & 0.917 & 0.00415 & 32.8 & 21 & COREAD \\
\hline PLX-4720 & BRAF & 0.916 & 0.00636 & 14.2 & 20 & COREAD \\
\hline Olaparib & PARP1, PARP2 & -0.688 & 0.00835 & 33.4 & 25 & COREAD \\
\hline FH535 & PPARgamma, PPARdelta & -0.821 & 0.00864 & 21.2 & 21 & COREAD \\
\hline Tamoxifen & ESR1 & 0.755 & 0.0136 & 34 & 26 & COREAD \\
\hline JQ1 & BRD2, BRD3, BRD4, BRDT & 0.704 & 0.0155 & 60.9 & 26 & COREAD \\
\hline Ruxolitinib & JAK1, JAK2 & 0.724 & 0.0254 & 72.9 & 26 & COREAD \\
\hline WZ-1-84 & BMX & -0.96 & 0.0343 & 29.4 & 6 & COREAD \\
\hline
\end{tabular}

\section{Systems Biology approach to predict dynamic changes in KRAS Mutations}

It has been demonstrated through various studies that there is no such information available that could reveal the direct or indirect link between $K R A S$ and colon cancer, but here a hypothetical based biochemical pathway is proposed by considering the available literature survey and TCGA data. Hence, recent studies had reported that few mutations inhibit the activity of $K R A S$ and it is 


\section{Supporting Information}

observed that the proposed mutants are more significant and robust compared to the previously reported mutants which play a crucial role in the development of colon cancer.

There is a transmembrane protein which is called epidermal growth factor, acting as a receptor for members of the epidermal factor's family of extracellular ligands of proteins known as EGFR ${ }^{1}$. It is triggered by the binding of its particular ligands, including epidermal growth factor and transforming growth factor $\alpha(\mathrm{TGF} \alpha)^{2}$. ErbB2 has no known direct activating ligand, and maybe in an activated state constitutively or become active upon heterodimerization with other family members such as EGFR. Upon activation by its growth factor ligands, EGFR undergoes a transition from an inactive monomeric form to an active homodimer ${ }^{3}$ although, there is evidence that the preformed inactive dimers may also exist before the ligand binding. Additionally, EGFR may pair with another member of the ErbB receptor family, such as ErbB2/Her2/neu, to create an activated heterodimer. There is also evidence to suggest that clusters of activated EGFRs are formed. However, it remains unclear whether this clustering is important for activation itself or occurs after the activation of an individual dimer. The reduced signaling of EGFR may result in the development of Alzheimer's while its overexpression results in the formation of various tumors ${ }^{4}$.

The KRAS quality gives direction to making a protein called K-Ras that is a piece of a flagging pathway known as the RAS/MAPK pathway. The KRAS quality has a place with a class of qualities known as oncogenes. Whenever changed, oncogenes can make ordinary cells lose their native function and may become cancerous. A group of qualities that make proteins engaged with cell flagging pathways, controlling cell development and cell demise. Transformed types of RAS quality might be found in a few sorts of diseases. These progressions may make malignant growth cells develop and spread in the body. Oncogenes are qualities that are firmly connected to the disease, and the quality that encodes Ras was among the first to be found. Ras is associated with the signals that control the measure of development that has to be permitted. Malignant growth causing the change of Ras makes a type of protein that is consistently active. It enables the cell to develop and separate. A couple of cancer syndromes are caused by the acquired transformations of proto-oncogenes that is a reason for turned on (initiated). Ras, a little GTP-restricting protein, is a vital part of the flag transduction pathway utilized by the developmental components to start cell growth and separation. Cell actuation with the developmental factors, for example, epidermal development factor (EGF) instigates Ras to move from an idle GDP-bound state to a functioning GTP-bound state. A remote-access administration (RAS) is any blend of equipment and programming to empower the remote access apparatuses or data that ordinarily dwell on a system of IT gadgets. A remote-access benefit interfaces a customer to a host PC, known as a remote access server.

The actuation of a proto-oncogene into an oncogene can happen by point transformation, quality intensification, and quality translocation. The primary human oncogene to be recognized encodes a constitutively dynamic type of Ras, a flag transduction protein. This oncogene was segregated from a human bladder carcinoma. The PI3K/AKT/mTOR pathway is an intracellular flagging pathway that is imperative in controlling the cell cycle ${ }^{6}$. Along these lines, it is directly identified with cell peacefulness, expansion, malignant growth, and life span. PI3K enactment phosphorylates and initiates AKT, restricting it in the plasma film.

One of the human genes known as BRAF is used for encoding a protein known as B-Raf ${ }^{7}$. This type of protein is also referred to as the proto-oncogene B-Raf and also as V-Raf murine viral oncogene homolog B but more formally, it is known as serine protein kinases, B-Raf. MEK 


\section{Supporting Information}

remains for the MAPK/ERK kinase, and it is among the arrangement of administrative kinases focusing on that pathway. It has a double job, going about as both a serine/threonine kinase and a tyrosine kinase. Mitogen-initiated protein kinase (otherwise called MAP2K, MEK, MAPKK) is a kinase chemical which phosphorylates mitogen-enacted protein kinase (MAPK). MAP2K is a delegated EC. Mitogen-enacted protein kinases (MAPKs) are an exceptionally monitored group of serine/threonine protein kinases that are associated with an assortment of basic cell procedures, for example, expansion, separation, motility, stretch reaction, apoptosis, and survival.

Mitogen-initiated protein kinase 1 (MAPK1) is also known as "extracellular flag directed kinase 2" (ERK2). Two comparative (85\% succession personality) protein kinases were initially called ERK1 and ERK2. For a proto-oncogenic translation factor of the bZIP family, dimerizes with proteins of the JUN family, in this manner framing the interpretation factor complex AP-1. FOS proteins work as controllers of cell multiplication, separation, and change.

One more factor which is known as TGFB in the transforming growth factor-beta is used for flagging the pathways and it is involved in different cellular processes in both of the organism's cell and development, differentiation of cell, apoptosis, and some kind of cell capacities. This protein is found in all tissues but is more abundant in bones, lungs and placental tissues such as the kidney.

TGF-beta ${ }^{8}$ is created by numerous yet not all parenchymal cell types and is likewise delivered or discharged by invading cells such as lymphocytes, monocytes/macrophages, and platelets. The TGF-beta flagging has a dual nature, acting both as a tumor silencer and tumor advertiser. On numerous occasions, this obstruction is a piece of a flagging switch whereby TGFbeta loses its development inhibitory impacts which are later utilized by the epithelial cell in an advanced way for the progression. Mitogen-initiated protein kinase 7 (MAP3K7), otherwise called TAK1, is a compound that in people is encoded by the MAP3K $7^{9}$ quality. The c-Jun NH2termina $1^{10}$ kinases (JNKs) are a gathering of mitogen-initiated protein (MAP) kinases that take part in the flag transduction occasions interceding particular cell capacities. Enactment of JNK is directed by phosphorylation in the light of cell push and provocative cytokines.

Table S2. : The concentration quantity and essential parameters for the $K R A S$ and related species during the time course simulation.

\begin{tabular}{|l|l|l|l|l|l|l|l|}
\hline ID & Name & Compartment & $\begin{array}{l}\text { Quantity } \\
\text { Type }\end{array}$ & $\begin{array}{l}\text { Initial } \\
\text { Quantity }\end{array}$ & Value & $\begin{array}{l}\text { Boundary } \\
\text { Condition }\end{array}$ & Constant \\
\hline s1 & KRAS & c1 & Concentration & 0.5 & 0.1 & false & true \\
\hline s2 & EGFR & c1 & Concentration & 0.5 & 0.1 & false & true \\
\hline s3 & PI3K & c1 & Concentration & 0.5 & 0.1 & false & true \\
\hline s4 & TGFb & c1 & Concentration & 0.5 & 0.1 & false & true \\
\hline s5 & MAPK & c1 & Concentration & 0.5 & 0.1 & false & true \\
\hline s6 & BRAF/Raf & c1 & Concentration & 0.5 & 0.1 & false & true \\
\hline s7 & MEK & c1 & Concentration & 0.5 & 0.1 & false & true \\
\hline s8 & ERK & c1 & Concentration & 0.5 & 0.1 & false & true \\
\hline s9 & FOS & c1 & Concentration & 0.5 & 0.1 & false & true \\
\hline
\end{tabular}


Supporting Information

\begin{tabular}{|l|l|l|l|l|l|l|l|}
\hline $\mathrm{s} 10$ & JUN & $\mathrm{c} 1$ & Concentration & 0.5 & 0.1 & false & true \\
\hline $\mathrm{s} 11$ & JNK & $\mathrm{c} 1$ & Concentration & 0.5 & 0.1 & false & true \\
\hline $\mathrm{s} 12$ & TAK1 & $\mathrm{c} 1$ & Concentration & 0.5 & 0.1 & false & true \\
\hline
\end{tabular}
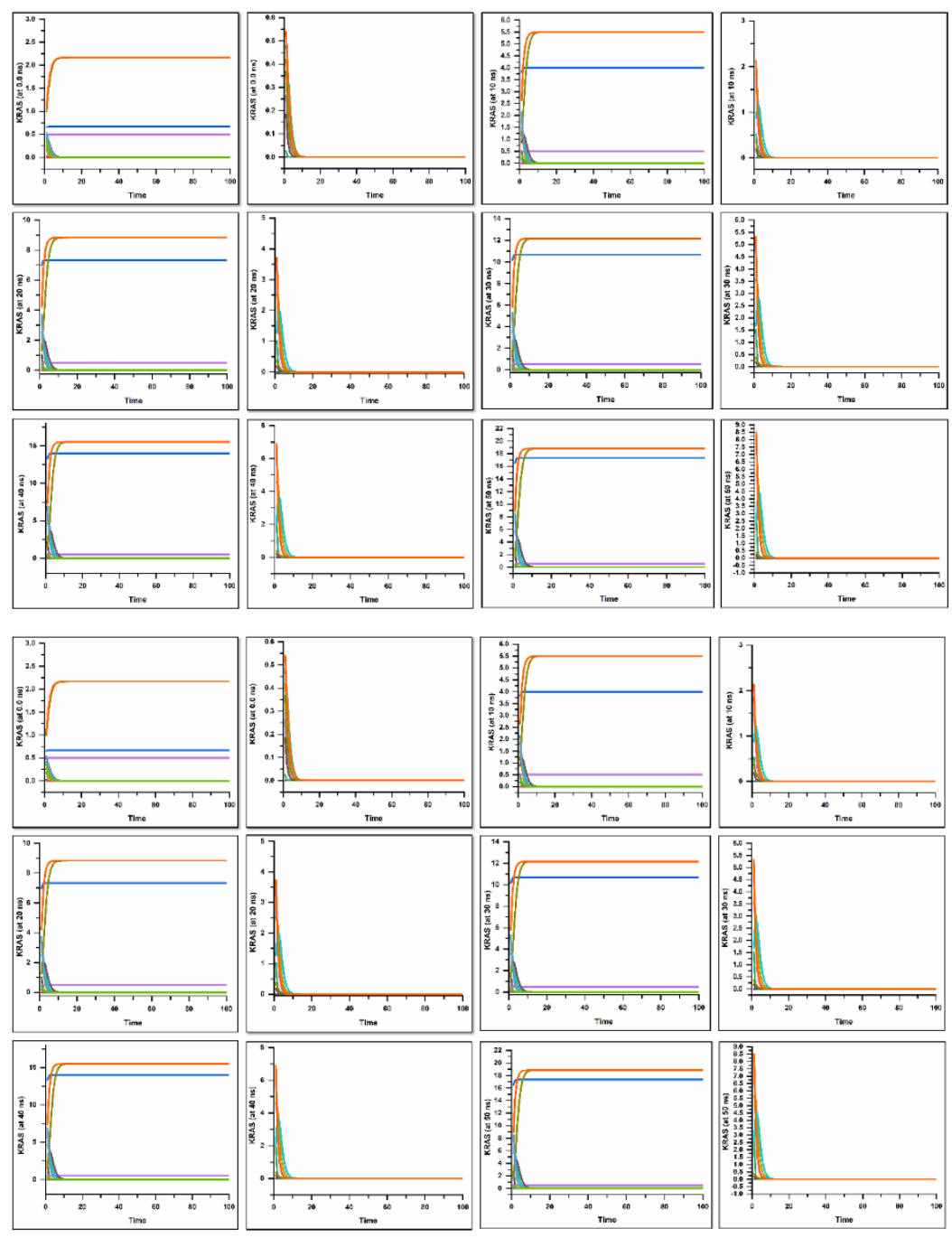

Figure S1. This graph indicates the relation between entities with proposed mutants. This study depicts that the few mutations inhibit the activity of $K R A S$ and we observed proposed mutants are more significant and robust as compared to the previously reported mutants which play a crucial role in colon cancer. 


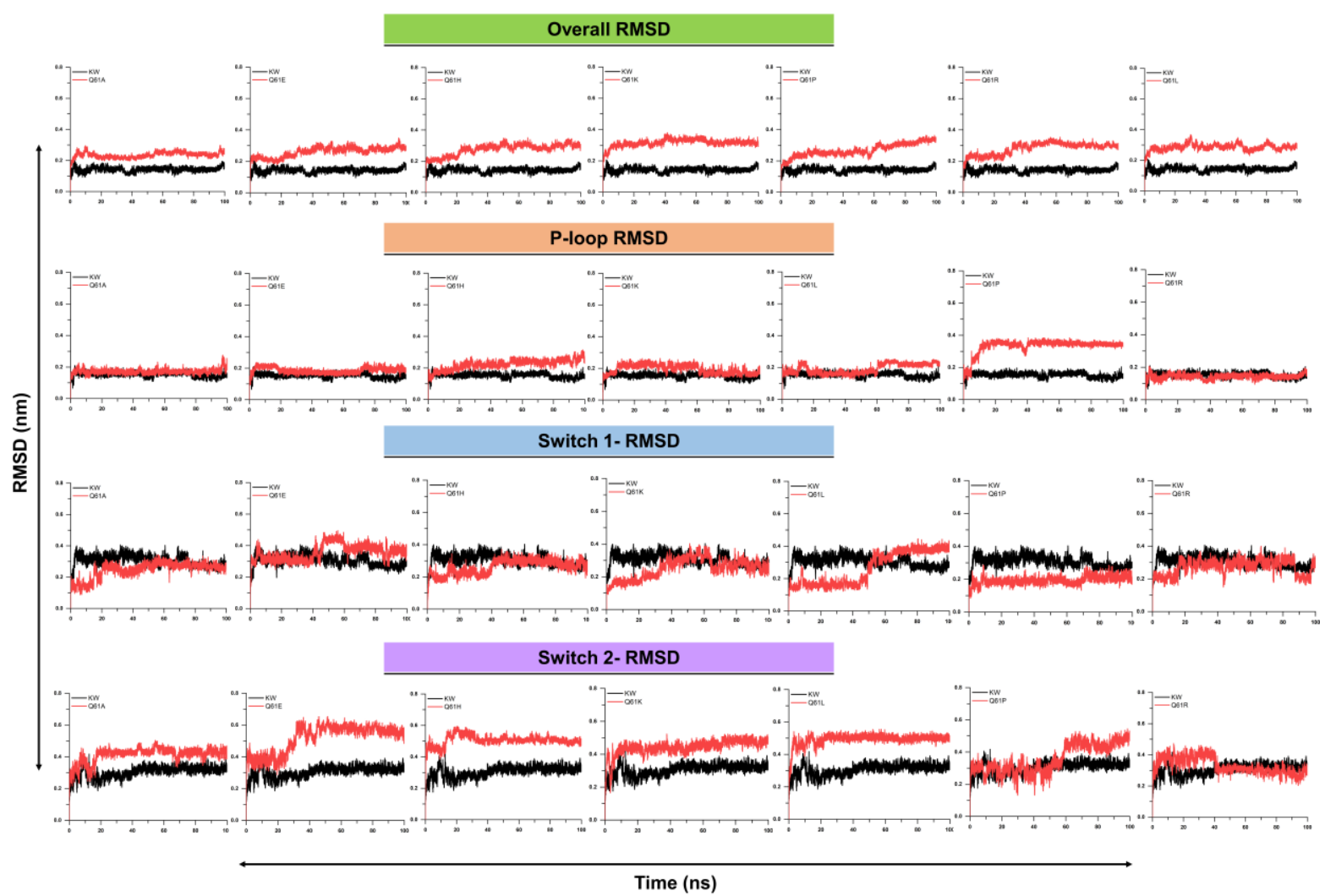

Figure S2. The RMSD graphs of the KRAS WT and mutants regarding its P-loop, Switch-I region, Switch-II region and the overall RMSD are individually drawn for detailed inspection. For a cobined visual, please refer to the main file (Figure 9). 


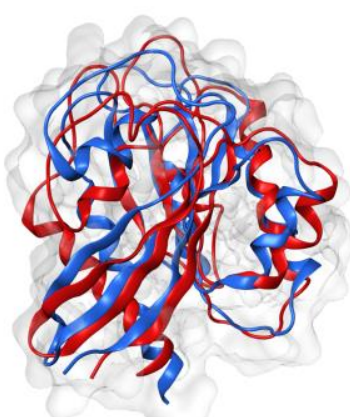

WT-Q61A

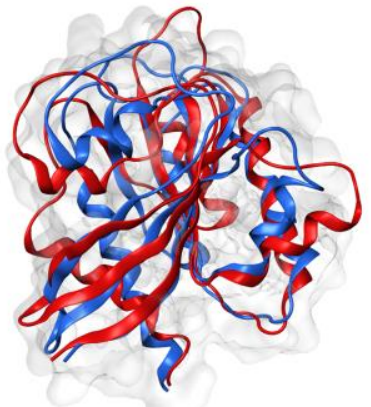

WT-Q61E

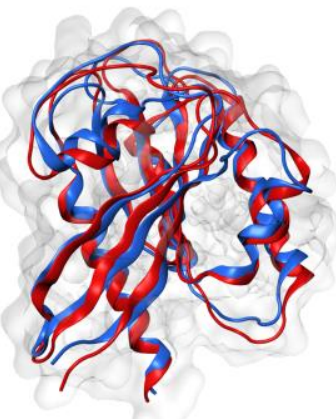

WT-Q61H

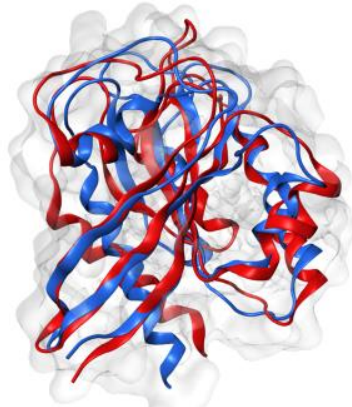

WT-Q61K

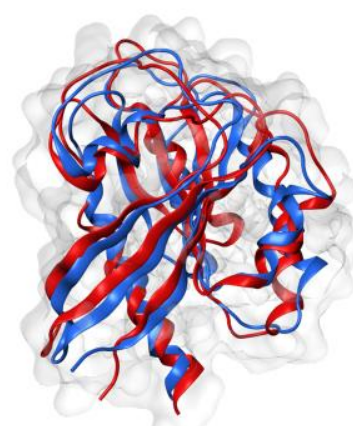

WT-Q61L

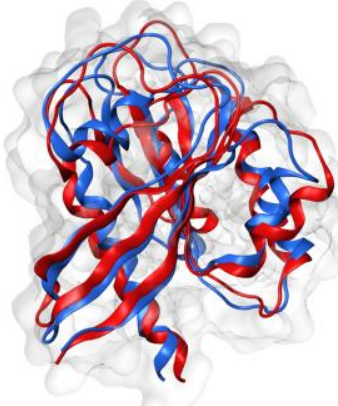

WT-Q61P

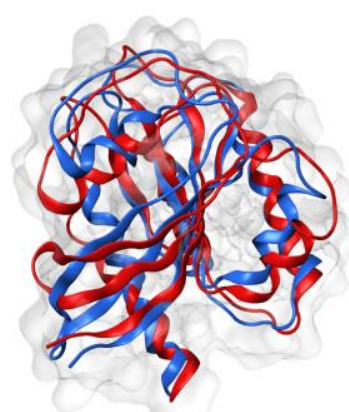

WT-Q61R

Figure S3. Superimposition of the KRAS WT and codon 61 mutants. A clear deviation from the native structure can be observed throughout the structure with higher fluctuation in the loop regions that are the sensitive sites of the protein.

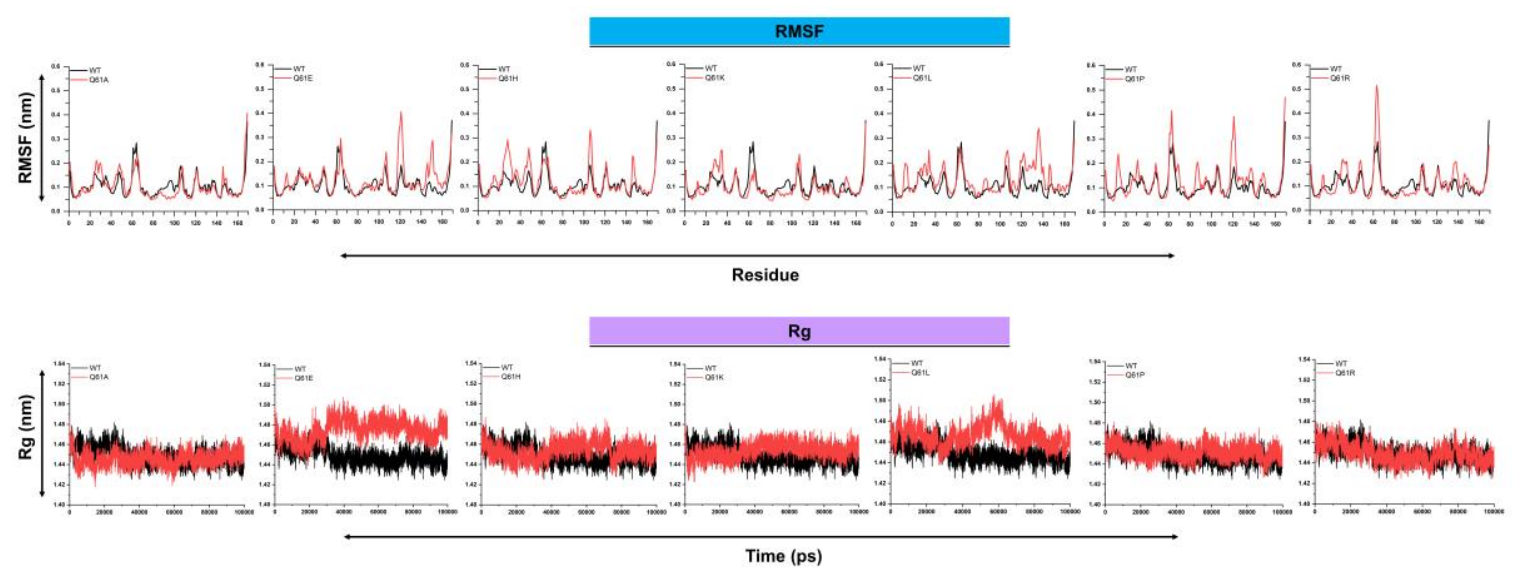

Figure S4. The root mean square fluctuation (RMSSF) and radius of gyration (Rg) graphs of the KRAS WT drawn individually against the codon 61 mutations. For combined visual inspection, please refer to the main article (Figure 11). 


\section{Supporting Information}

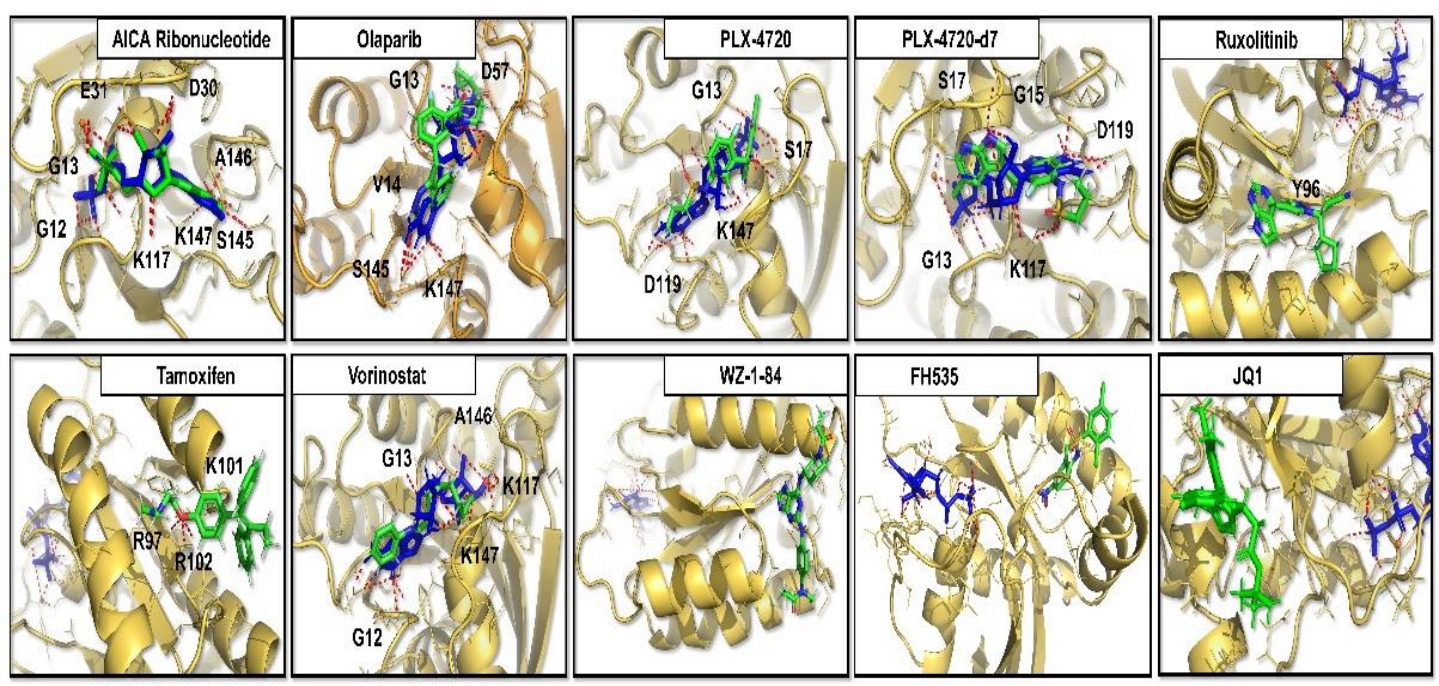

Figure S5. FDA approved drugs interacting with the $K R A S$.

The KRAS 3D structure was set by removing all the water molecules. The protonation was done and also the Kollman charges were assigned. The Powell's approach was used for the energy minimization the PDB structure was converted into the pdbqt format. The grid points were set to $60 \times 60 \times 60 \AA$, with the important sites locating at the grid box's center. The Lamarckian genetic algorithm in Autodock 4.0. ${ }^{47-49}$ was used for the estimation of binding potential with a spacing set to $0.375 \AA^{50}$. The parameters used were $300,0.2$ and 0.8 for the population size, mutation rate, and crossover respectively. The docking simulations were carried out with energy evaluations kept as 1,500,000 and maximum generations allowed up to 27000. A total of 20 docked conformations for each compound were yielded as each simulation was repeated 20 times. The binding energies between a receptor and a ligand were calculated over a grid map that is generated by the AutoGrid tool $^{51}$. The minimum energy conformations were considered as the binding conformations among the drug compounds and the proteins. For exploring the protein-ligand interactions, the PyMOL software was used ${ }^{52}$. 
Supporting Information

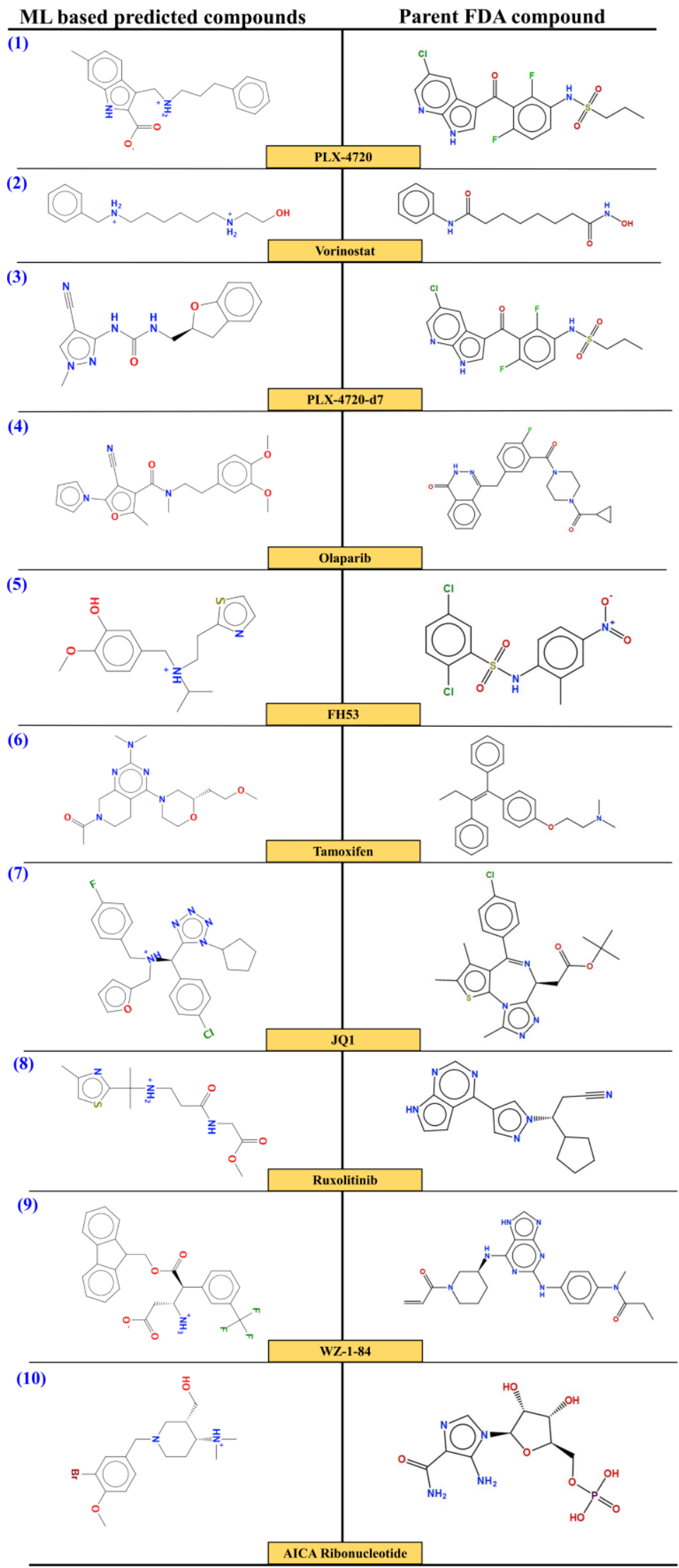




\section{Supporting Information}

Figure S6: Top ten compounds predicted via a machine learning approach that shows better affinity as compared to the FDA approved drugs with the target for colon cancer. The parent FDA compounds are also given for side-by-side comparision.

\section{Chapter 2}

\section{Description of the features used}

Features considered in our work are composition vector, composition moment vector, basic features, neighborhood index, R group, electronic group, hydrophobic, and side chains.

Their description is given below.

\section{(i) Composition vector:}

CVi denotes the percentage composition of amino acid (AA) $\mathrm{i}$ in the protein sequence: $\mathrm{CVi}=$ (number of amino acid $\mathrm{i}$ in the sequence)/(total number of AA's in the sequence). 20 amino acids were coded in alphabetical order: A, C, D, E, F, G, H, I, K, L, M, N, P, Q, R, S, T, V, W, Y, and were denoted AA1, AA2, .., AA20, respectively.

The reference for composition vector is https://doi.org/10.1186/1471-2105-7-518.

\section{(ii) Composition moment vector}

The composition moment vector of a protein was defined as follows:

For $\mathrm{k}=1, x_{i}^{(1)}$ the $\mathrm{i}$-th entry of the frst-order composition moment vector,

$$
C M V_{i}^{1}=x_{i}^{(1)}=\frac{1}{N(N-1) \ldots(N-k)} \sum_{j}\left(n_{i j}\right)^{1}
$$

For $\mathrm{k}=2, x_{i}^{(1)}$ the $\mathrm{i}$-th entry of the second-order composition moment vector,

$$
C M V_{i}^{2}=x_{i}^{(2)}=\frac{1}{N(N-1) \ldots(N-k)} \sum_{j}\left(n_{i j}\right)^{2}
$$

Where $C M V$ is the $\mathrm{i}$-th $\mathrm{AA}$ in the sequence, $\mathrm{N}$ is the length of the sequence, $n_{i j}$ is the $\mathrm{j}$-th position of $\mathrm{AAi}$ and $\mathrm{k}$ is the order of the composition vector.

The reference for composition moment vector is https://doi.org/10.1002/prot.21527.

\section{(iii) Basic features or basic physiochemical features}

In this study, seven physicochemical properties were chosen from AA index. Tey include hydrophobicity, charge, polarity, volume, flexibility, isoelectric point, and refractivity. For each 


\section{Supporting Information}

of these properties, the basic physicochemical feature is calculated by $B P C=\sum_{i=1}^{N} P_{i}$, where $P_{i}$ is the relevant physiochemical property of the $\mathrm{i}$-th amino acid in a given sequence.

The reference for the basic physiochemical features is DOI: 10.1039/C7EN00466D.

\section{(iv) Neighboring index}

Neighbourhood-based physicochemical features. The seven physicochemical properties in the NPC are the same as those in the BPC. For each property, the NPC feature considers the effect of the properties of its neighboring AA and is calculated as:

$$
N P C=\sum_{i-1}^{N} \mid\left(P_{i}\right)^{2}-P_{i-1} * P_{i+1},
$$

where $\mathrm{N}$ is the length of the protein sequence, and $P_{i}$ is the concerned physicochemical property of the i-th amino acid in the sequence. The reference for the basic physiochemical features is https://doi.org/10.1038/s41598-019-43125-6.

\section{(iv) $\mathbf{R}$ group features}

There are five types of protein $R$ groups. $R G_{i}$ is the percentage of all amino acids in the sequence that have $R$ groups of type $i$, where $i=1,2, \ldots, 5$. The case of $i=1$ corresponds to nonpolar aliphatic AAs (A, G, I, L, M, V), i=2 to polar uncharged AAs (C, N, P, Q, S, T), $i=3$ to positively charged AAs $(\mathrm{H}, \mathrm{K}, \mathrm{R}), i=4$ to negative AAs $(\mathrm{D}, \mathrm{E})$, and $i=5$ to aromatic AAs $(\mathrm{F}, \mathrm{W}$, $\mathrm{Y})$. The reference is https://doi.org/10.1038/s41598-019-43125-6.

\section{(v) Electronic group features}

EleGi is the percentage composition of electronic group $i$ in the sequence, where $i=1,2$, $\ldots, 5$. The case in which $i=1$ corresponds to electron donor AAs (A, D, E, P), $i=2$ to weak electron donor AAs (I, L, V), $i=3$ to electron acceptor AAs $(\mathrm{K}, \mathrm{N}, \mathrm{R}), i=4$ to weak electron acceptor AAs $(\mathrm{F}, \mathrm{M}, \mathrm{Q}, \mathrm{T}, \mathrm{Y})$, and $i=5$ to neutral AAs $(\mathrm{G}, \mathrm{H}, \mathrm{S}, \mathrm{W})$. The reference https://doi.org/10.1016/j.jtbi.2010.11.017.

\section{(vi) Hydrophobicity group features}

Hydrophobicity groups were formed according to the water-soluble side chains of amino acids. $H G_{i}$ is the percentage composition of hydrophobicity group $i$ in the sequence. The case $i=1$ corresponds to hydrophobic AAs (A, C, F, G, I, L, M, P, V, W, Y), $i=2$ to hydrophobic basic AAs $(\mathrm{H}, \mathrm{K}, \mathrm{R}), i=3$ to hydrophobic acidic AAs $(\mathrm{D}, \mathrm{E})$, and $i=4$ to hydrophobic polar with uncharged side chain AAs (N, Q, S, T). The reference is https://doi.org/10.1016/S1672-0229(06)60023-5.

\section{(vii) Side chain group features}

Side chain groups were based on the attributes of side chains including molecular weight, polarity, aromaticity, and charge. $S C G_{i}$ is the percentage composition of side chain group $i$ in the sequence. The case in which $i=1$ corresponds to tiny side chain AAs (A, G), $i=2$ to bulky side chain AAs (F, H, R, W, Y), $i=3$ to polar-uncharged AAs (D, E), $i=4$ to charged side chain AAs 


\section{Supporting Information}

(D, E, H, I, K, L, R, V), $i=5$ to polar side chain AAs (D, E, K, N, Q, R, S, T, W, Y), and $i=6$ to aromatic side chain AAs (F, H, W, Y). Although this feature and Feature 6 were both based on the $\mathrm{R}$ groups of amino acids, they are different in division criteria and biological meaning. The reference is https://doi.org/10.1038/s41598-019-43125-6.

\section{Chapter 3}

Table S3. List of all the docked compounds along witht their structures, docking scores and molecular interactions on a $2 \mathrm{D}$ plane.

\section{(a) PLX-4720-Similar}

\begin{tabular}{|c|c|c|c|}
\hline S.No. & Compound structure & Docking score & 2D Interaction pose \\
\hline 1 & & -11 & \\
\hline 2 & & -9.45 & $(-\infty)$ \\
\hline 3 & & -8.354 & 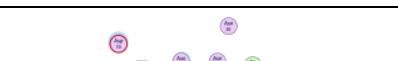 \\
\hline 4 & & -9.15 & \\
\hline 5 & & -18.038 & 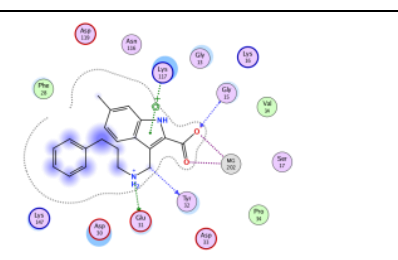 \\
\hline
\end{tabular}


Supporting Information

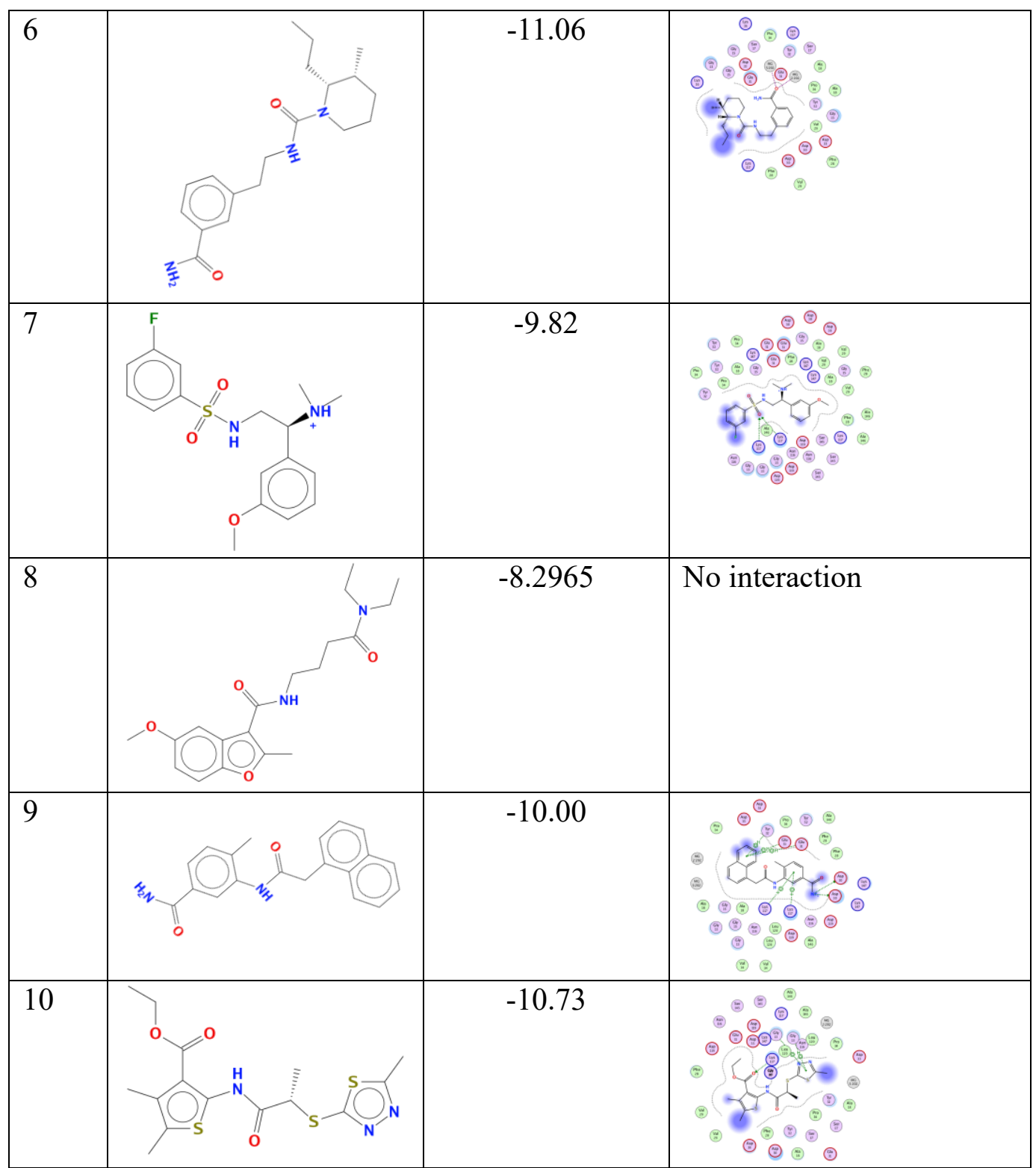

(b) Vorinostat-Similar

\begin{tabular}{|l|c|c|c|}
\hline S.No. & Compound structure & Docking score & 2D Interaction pose \\
\hline 1 & & -10.27 &
\end{tabular}


Supporting Information

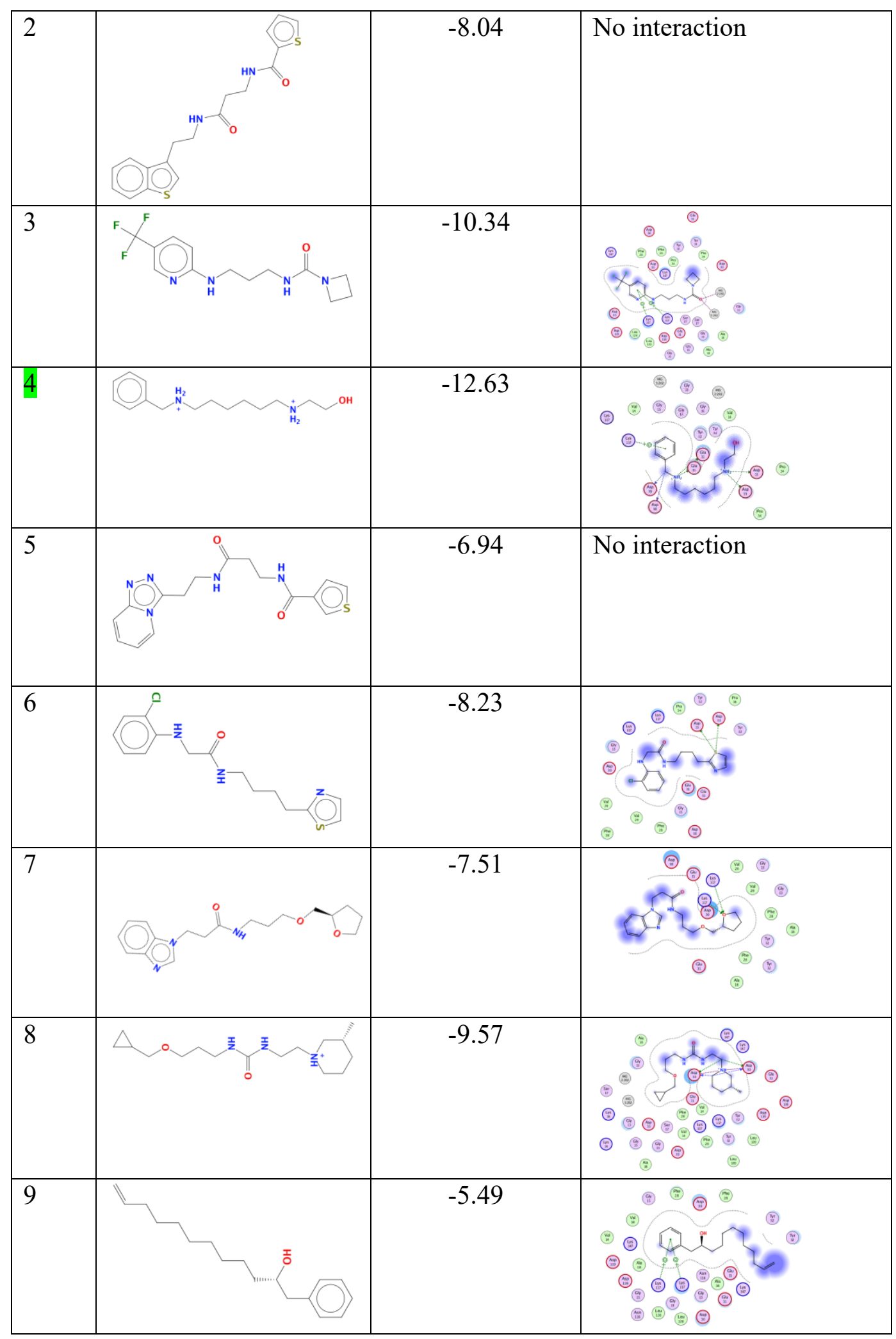


Supporting Information

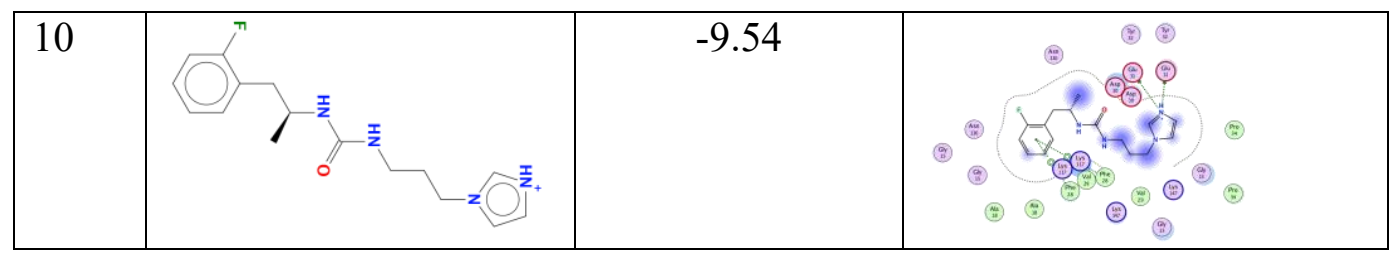

(c) PLX-4720-d7-Similar

\begin{tabular}{|c|c|c|c|}
\hline S.No. & Compound structure & Docking score & 2D Interaction pose \\
\hline 1 & & -9.41 & 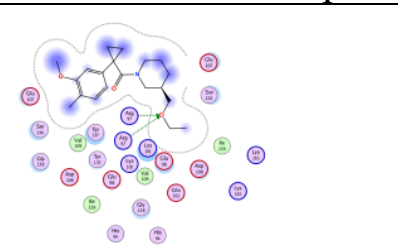 \\
\hline 2 & & -11.49 & 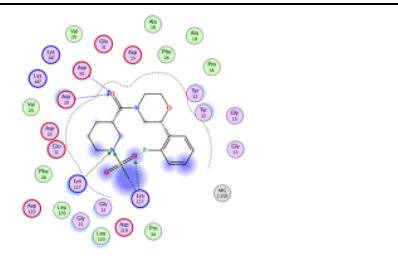 \\
\hline 3 & & -7.8 & No interaction \\
\hline 4 & & -11.38 & 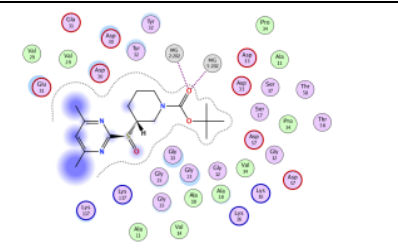 \\
\hline 5 & $\int_{N}-N$ & -14.06 & 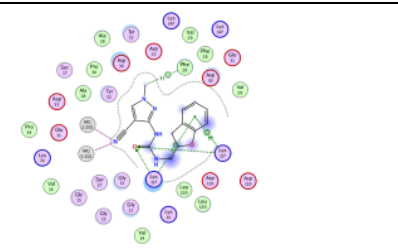 \\
\hline 6 & & -11.22 & 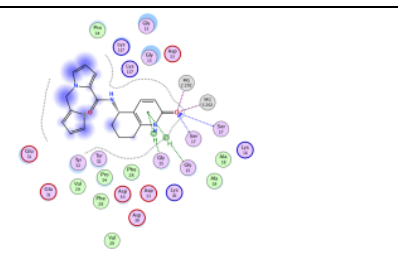 \\
\hline
\end{tabular}


Supporting Information

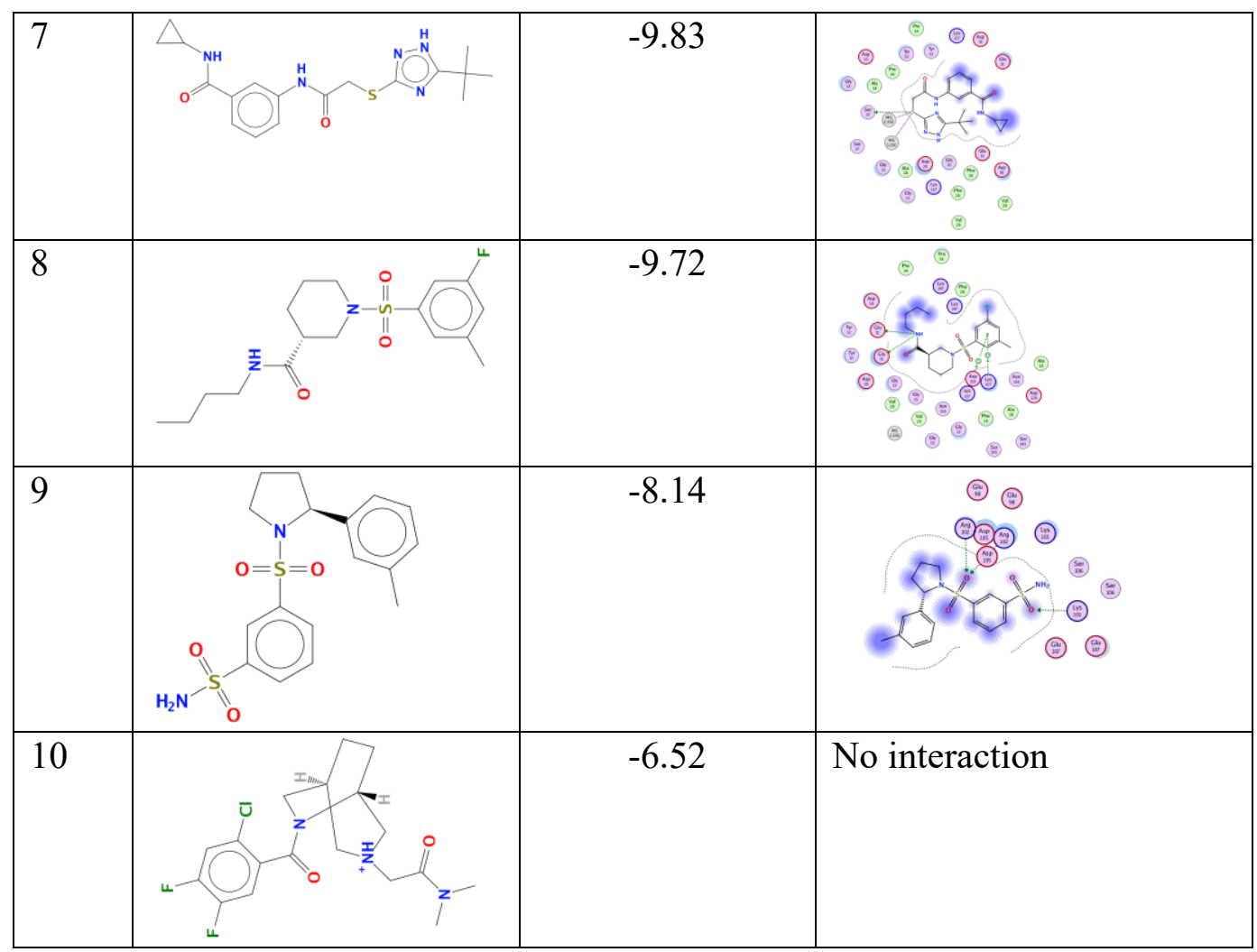

(d) Olaparib-Similar

\begin{tabular}{|l|c|c|c|}
\hline S.No. & Compound structure & Docking score & 2D Interaction pose \\
\hline 1 & & -10.23 & \\
\hline 2 & & & \\
\hline 3 & & &
\end{tabular}


Supporting Information

\begin{tabular}{|c|c|c|c|}
\hline 4 & $N_{0}$ & -11.71 & 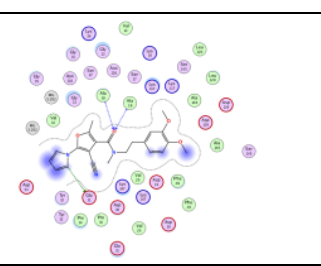 \\
\hline 5 & $\gamma^{-0} \sum_{\text {z- }}$ & -9.66 & 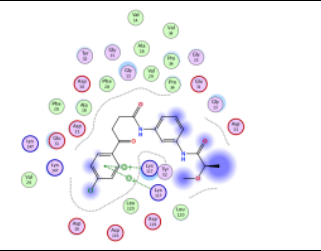 \\
\hline 6 & & -9.90 & 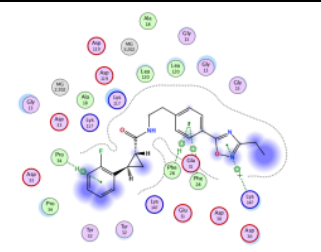 \\
\hline 7 & & -11.02 & 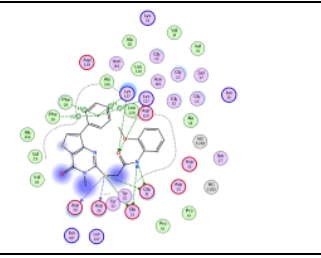 \\
\hline 8 & -0 & -10.87 & 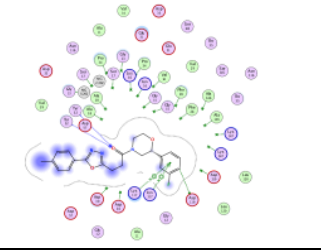 \\
\hline 9 & & -7.76 & 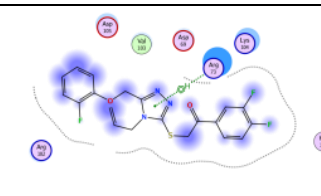 \\
\hline 10 & $\overline{0}$ & -8.35 & No interaction \\
\hline
\end{tabular}

(e) FH535-Similar

\begin{tabular}{|c|c|c|c|}
\hline S.No. & Compound structure & Docking score & 2D Interaction pose \\
\hline 1 & 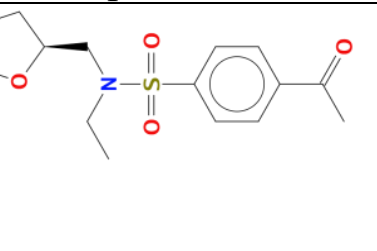 & -12.59 & 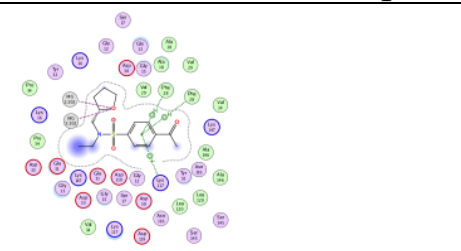 \\
\hline
\end{tabular}


Supporting Information

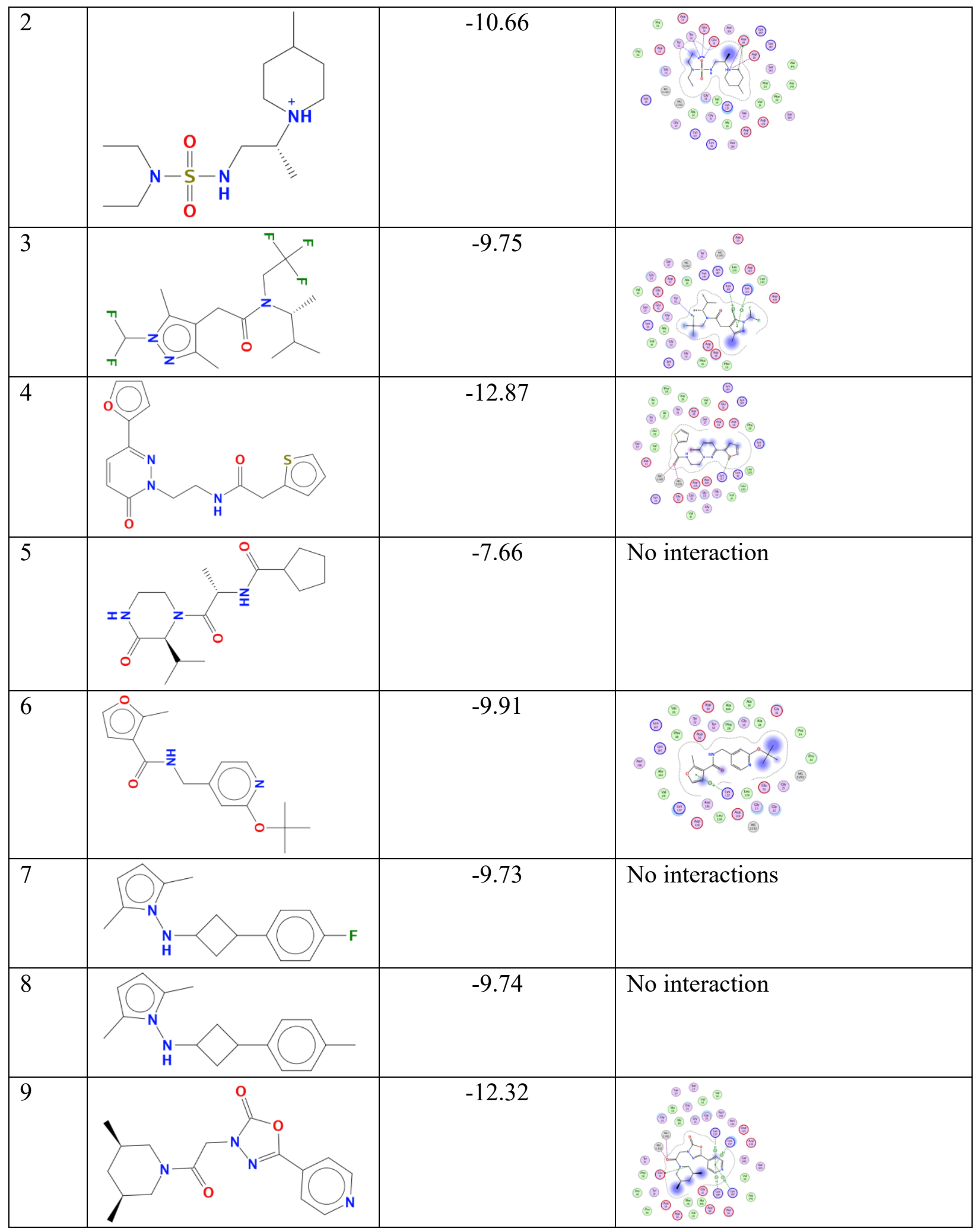


Supporting Information

\begin{tabular}{|l|l|l|l|}
\hline 10 & & -13.18 & \\
\hline
\end{tabular}

\section{(f) Tamoxifen-Similar}

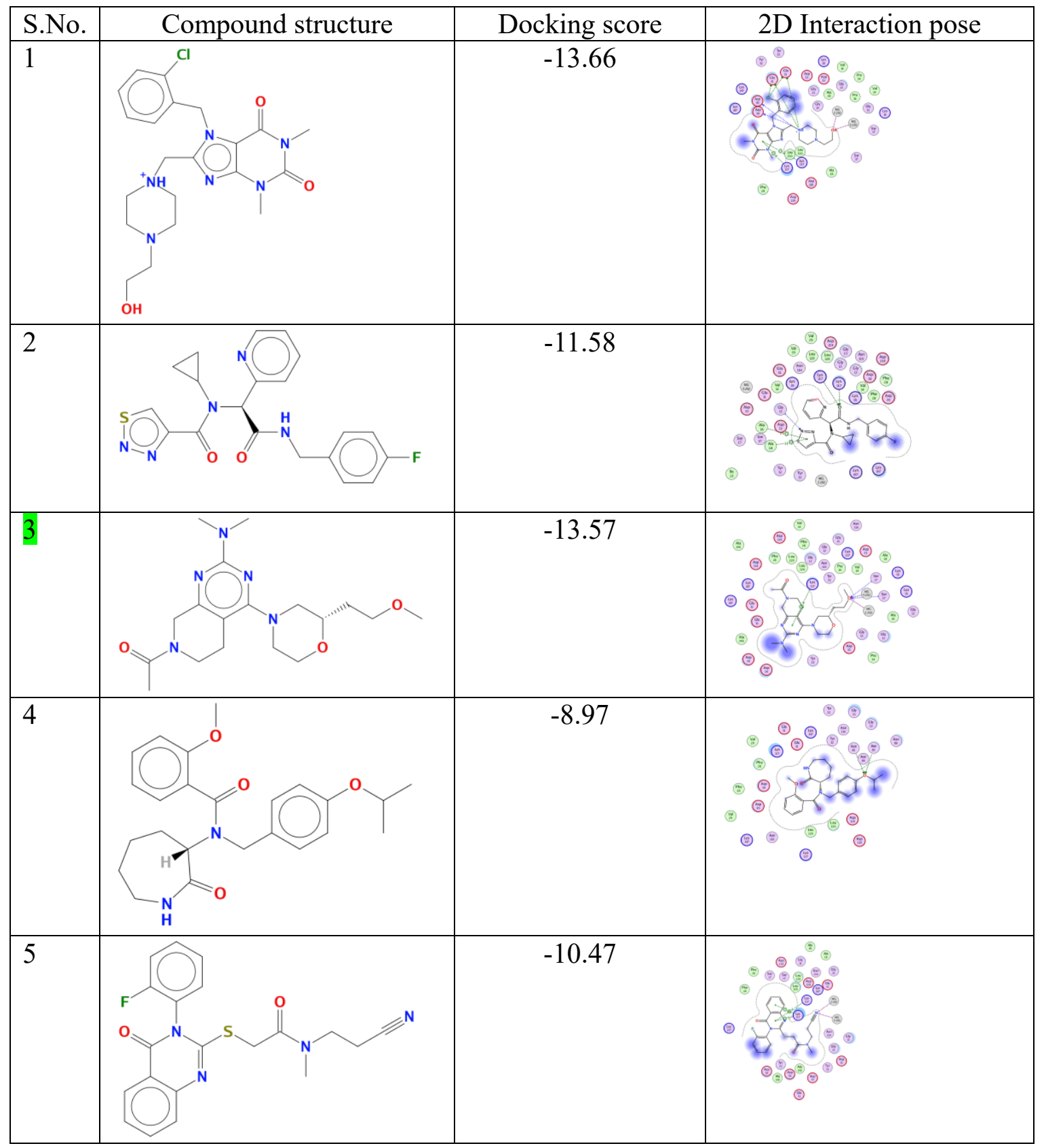


Supporting Information

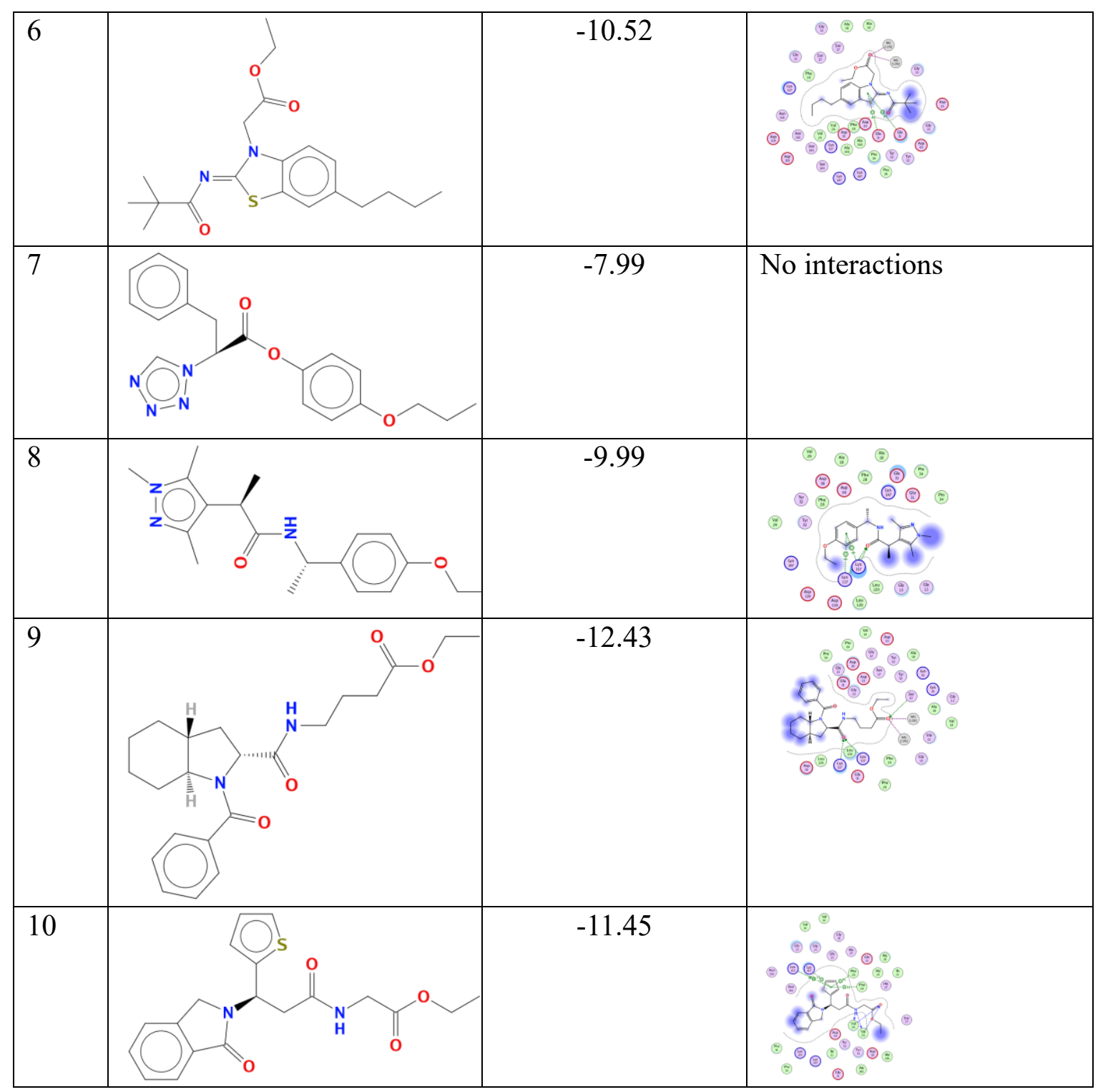

\section{(g) JQ1-Similar}

\begin{tabular}{|l|c|c|c|}
\hline S.No. & Compound structure & Docking score & 2D Interaction pose \\
\hline 1 & & -9.11 & \\
\hline
\end{tabular}


Supporting Information

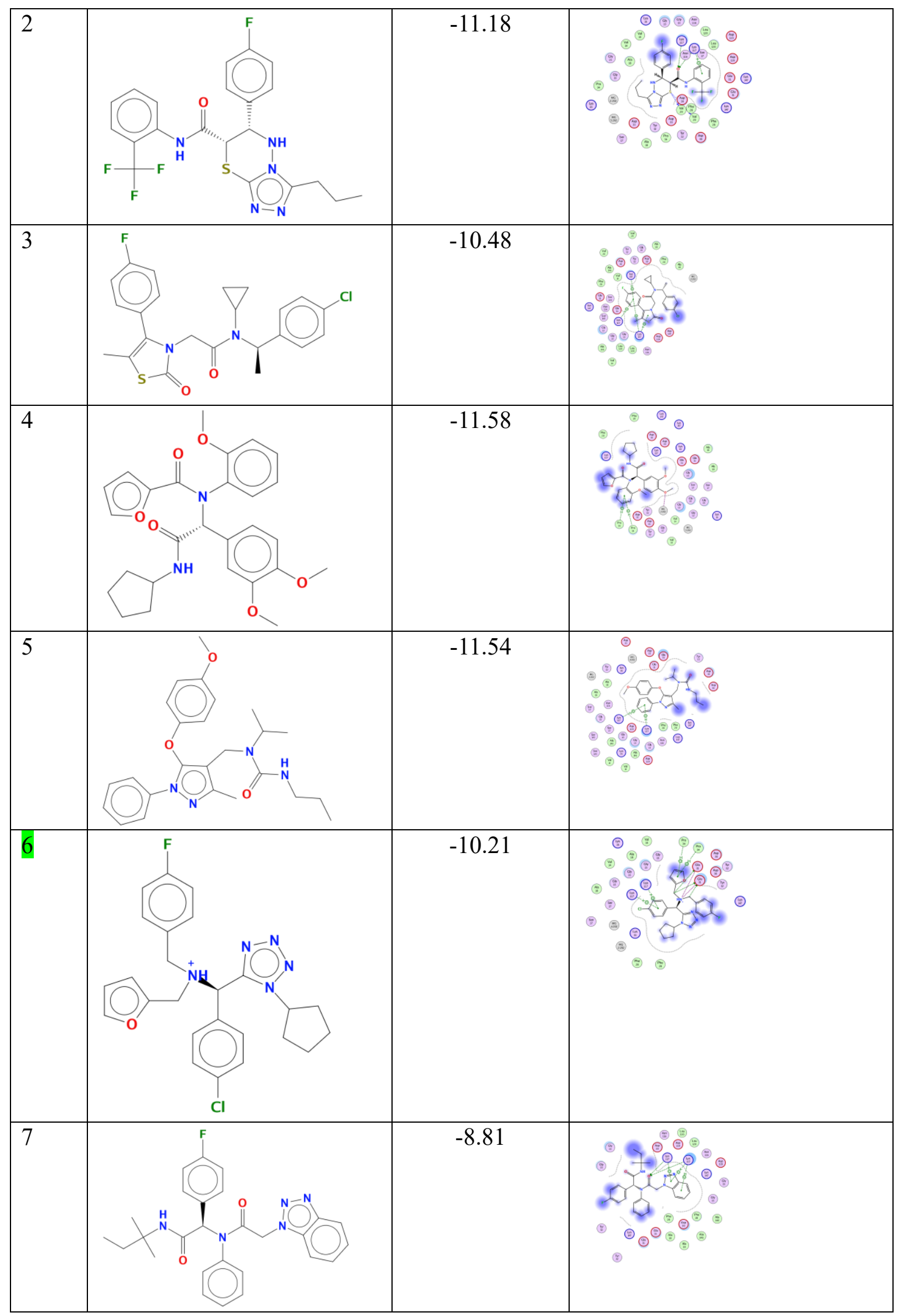


Supporting Information

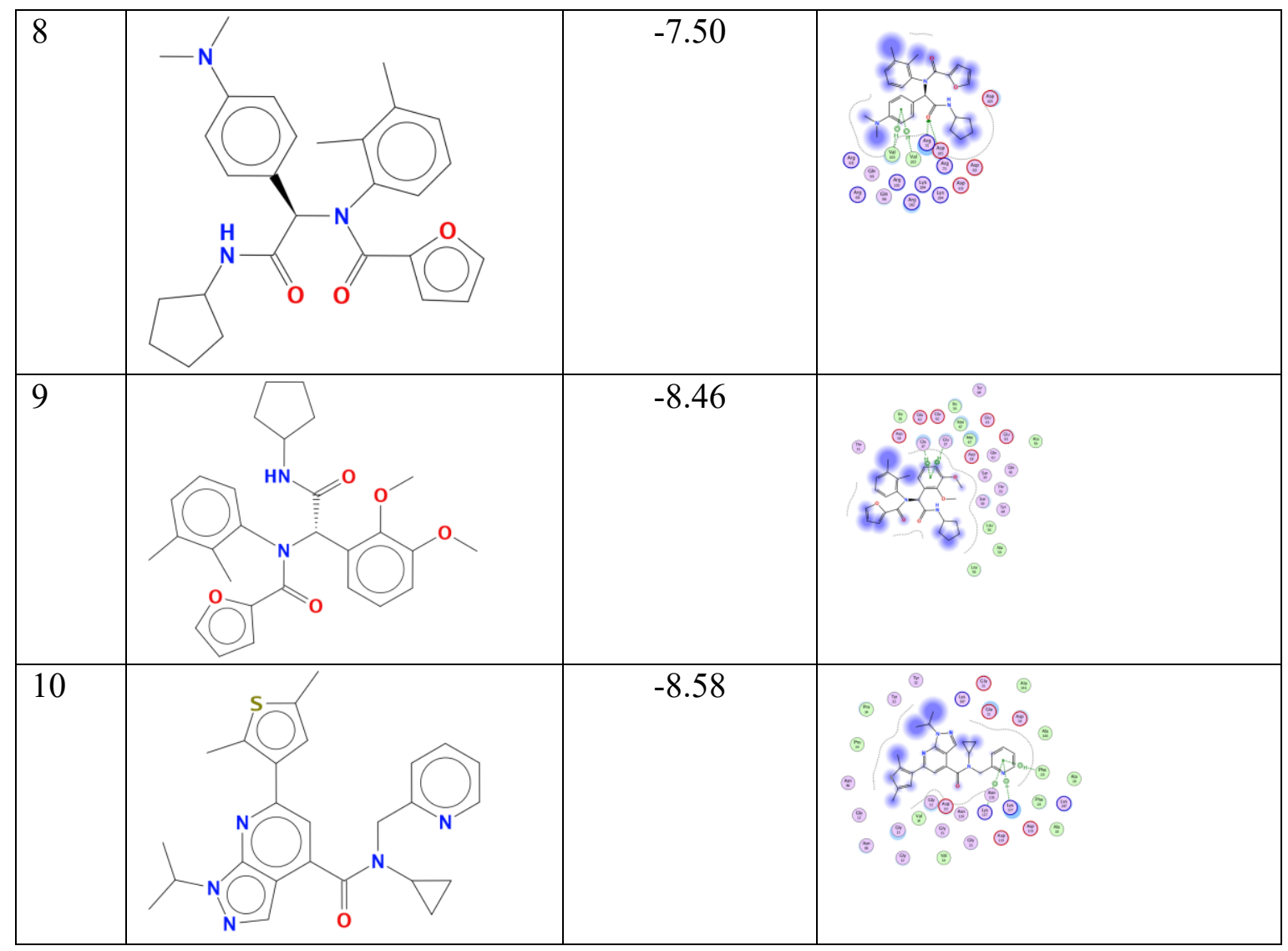

(h) Ruxolitinib-Similar

\begin{tabular}{|l|c|c|c|}
\hline S.No. & Compound structure & Docking score & 2D Interaction pose \\
\hline 1 & & -10.86 & \\
\hline 2 & & \\
\hline
\end{tabular}


Supporting Information

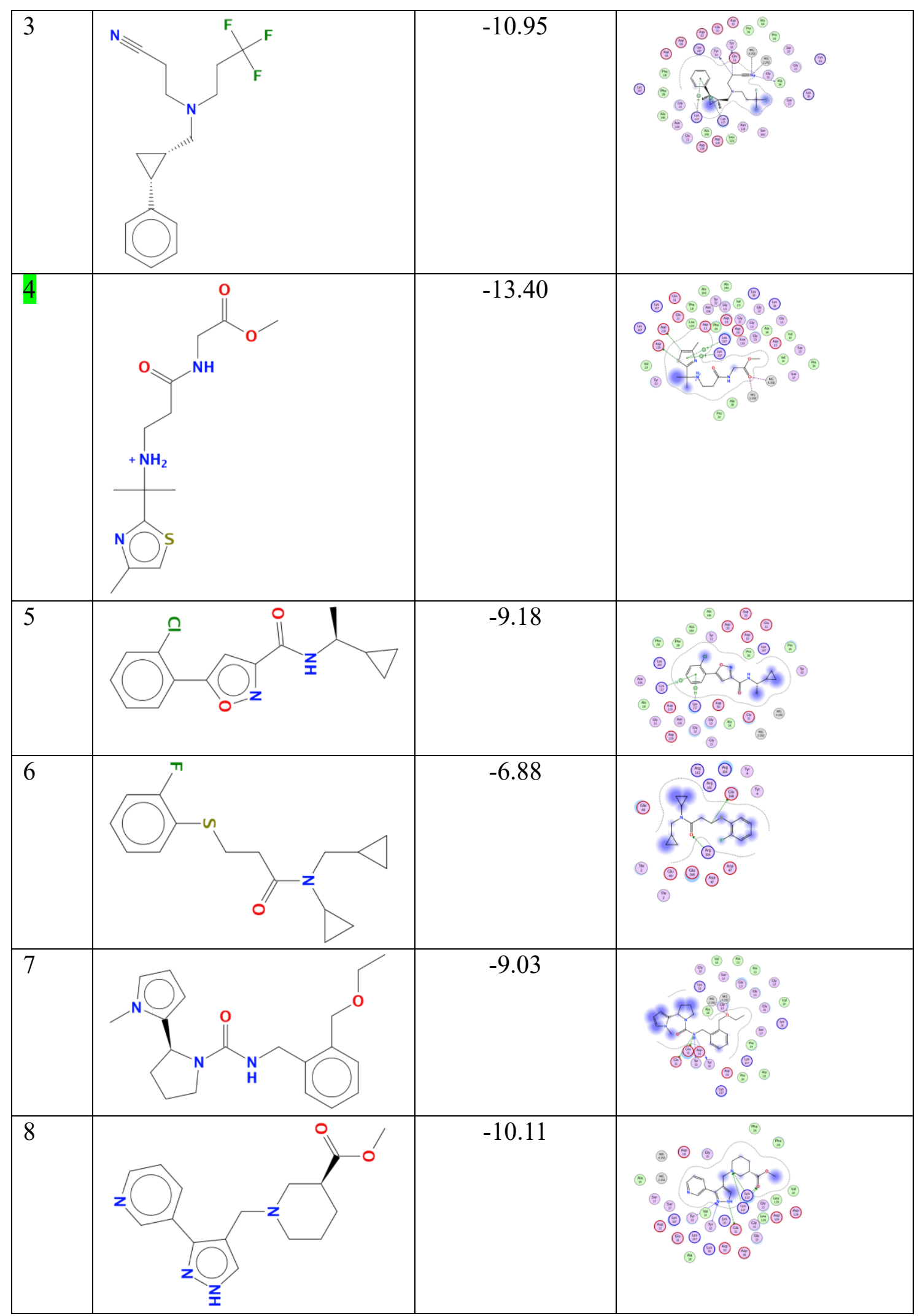


Supporting Information

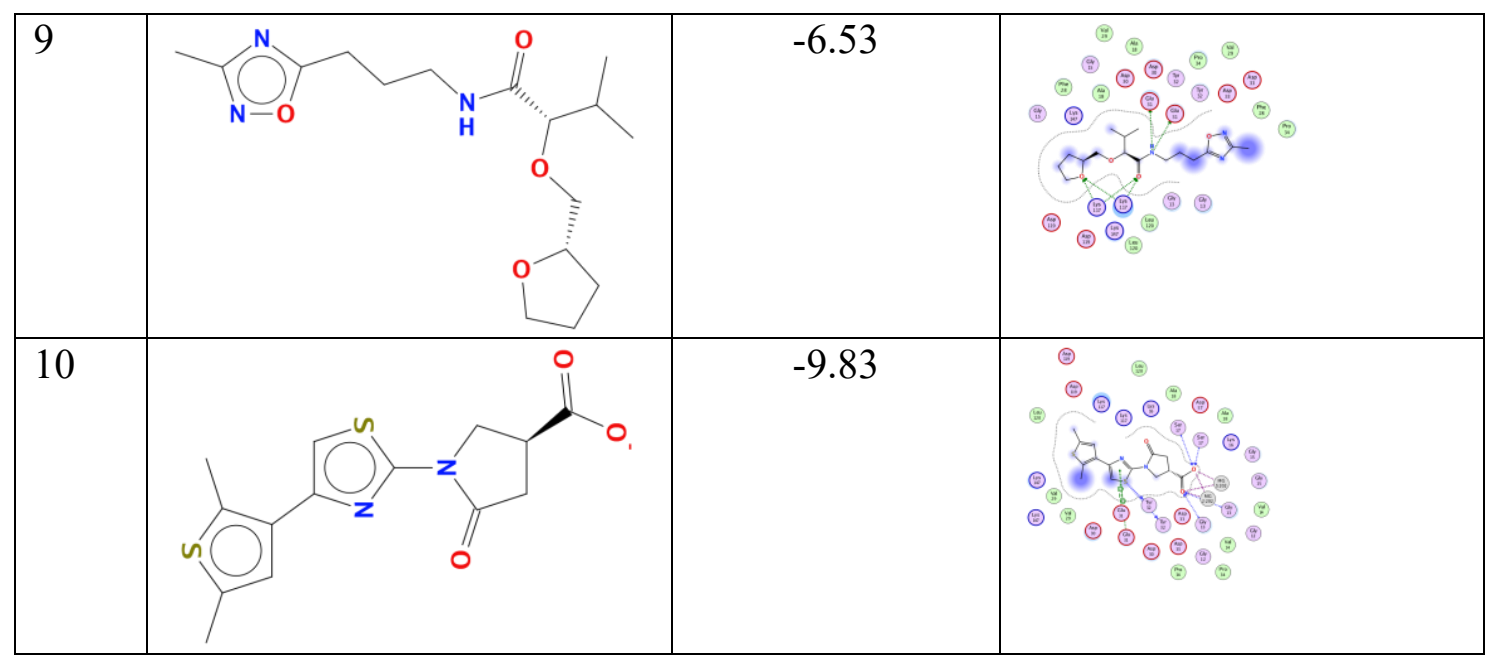

(i) WZ-1-84-Similar

\begin{tabular}{|l|c|c|c|}
\hline S.No. & Docking score & 2D Interaction pose \\
\hline 1 & -5.85 & \\
\hline 3 & -19.02 & \\
\hline 4 & & \\
\hline
\end{tabular}


Supporting Information

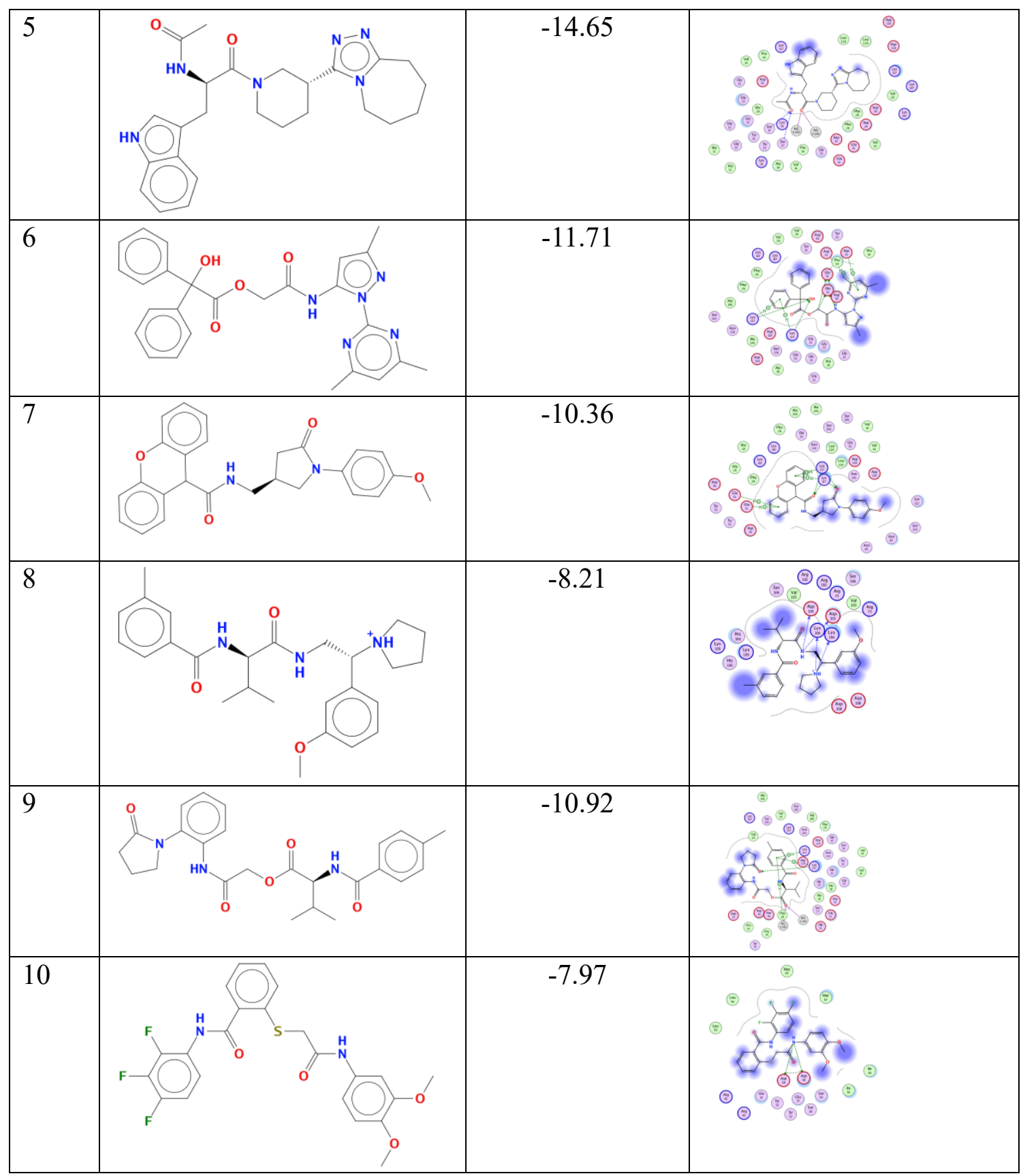

(j) AICA Ribonucleotide-Similar

\begin{tabular}{|l|c|c|c|}
\hline S.No. & Compound structure & Docking score & 2D Interaction pose \\
\hline 1 & & -10.28 & \\
& & & \\
\hline
\end{tabular}


Supporting Information

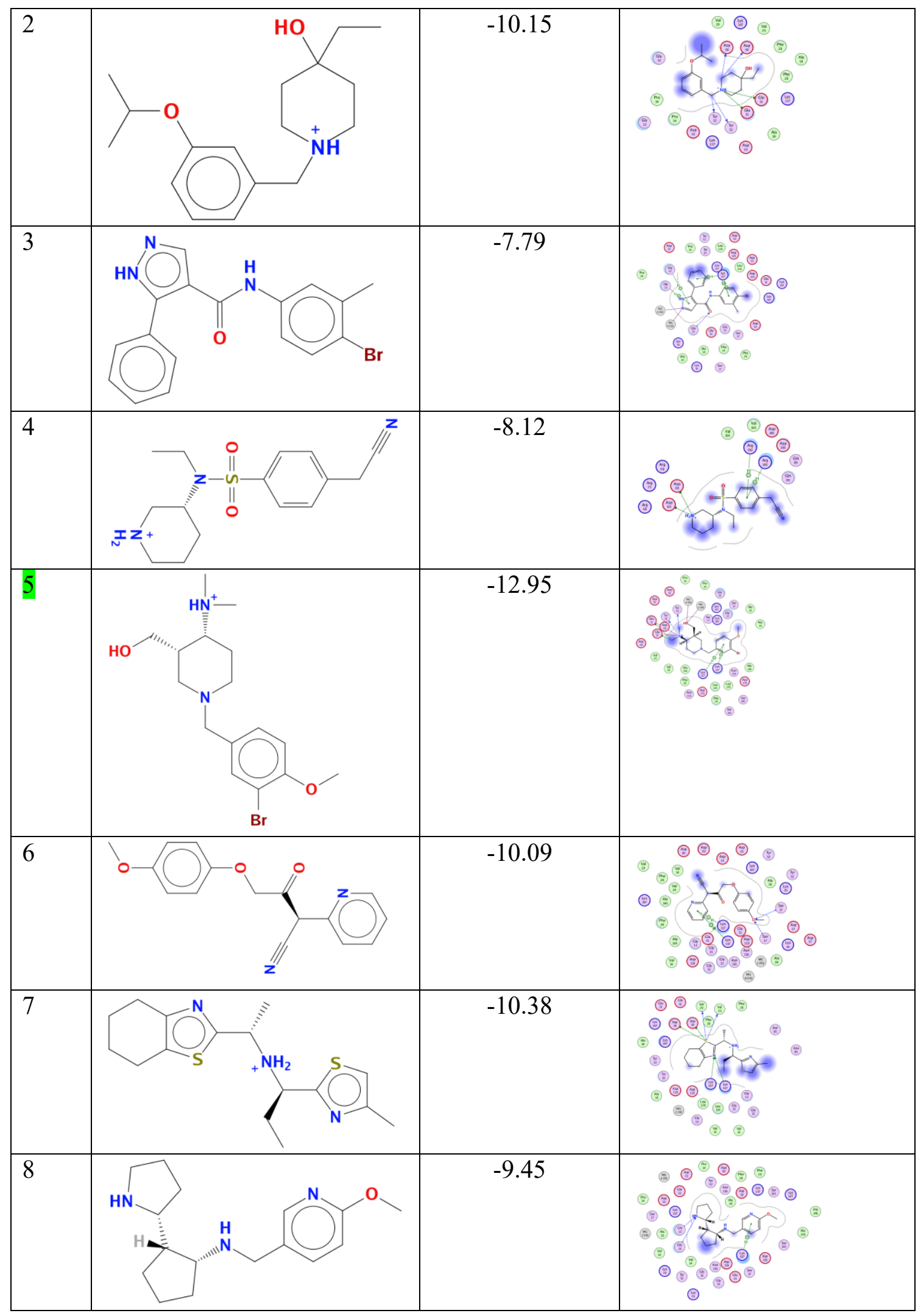


Supporting Information

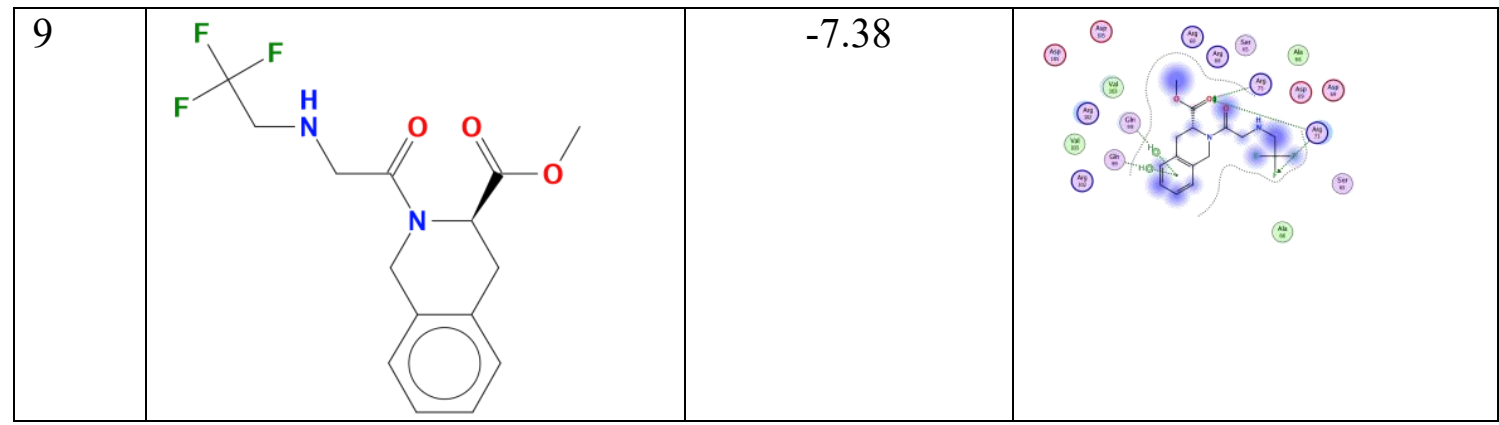

Table S4. Outcomes of the inverse docking approach where the top predicted compounds are individually docked to each mutated conformation of the KRAS protein, showing significant interactions and good docking scores.

\section{(i) AICA-Ribonucleotide-similar}

\begin{tabular}{|c|c|c|c|c|}
\hline S.No. & Compound str. & Docking score & Mutation & Interaction \\
\hline 1 & 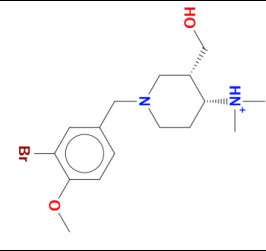 & -11.37 & Q61A & (3) \\
\hline 2 & J & -11.02 & Q61E & 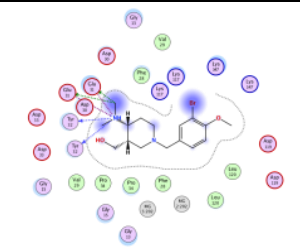 \\
\hline 3 & $\bar{I}$ & -10.63 & Q61H & 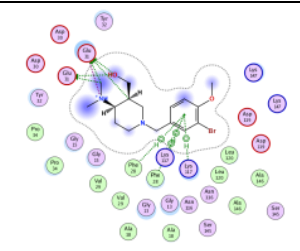 \\
\hline 4 & $\bar{I}$ & -9.45 & Q61K & 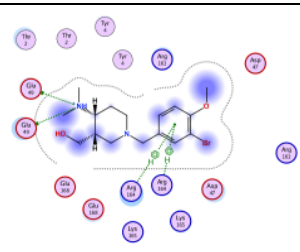 \\
\hline 5 & w & -11.43 & Q61L & 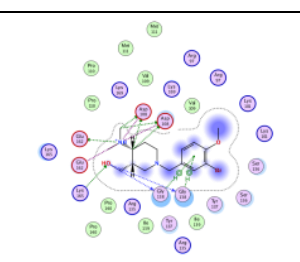 \\
\hline
\end{tabular}


Supporting Information

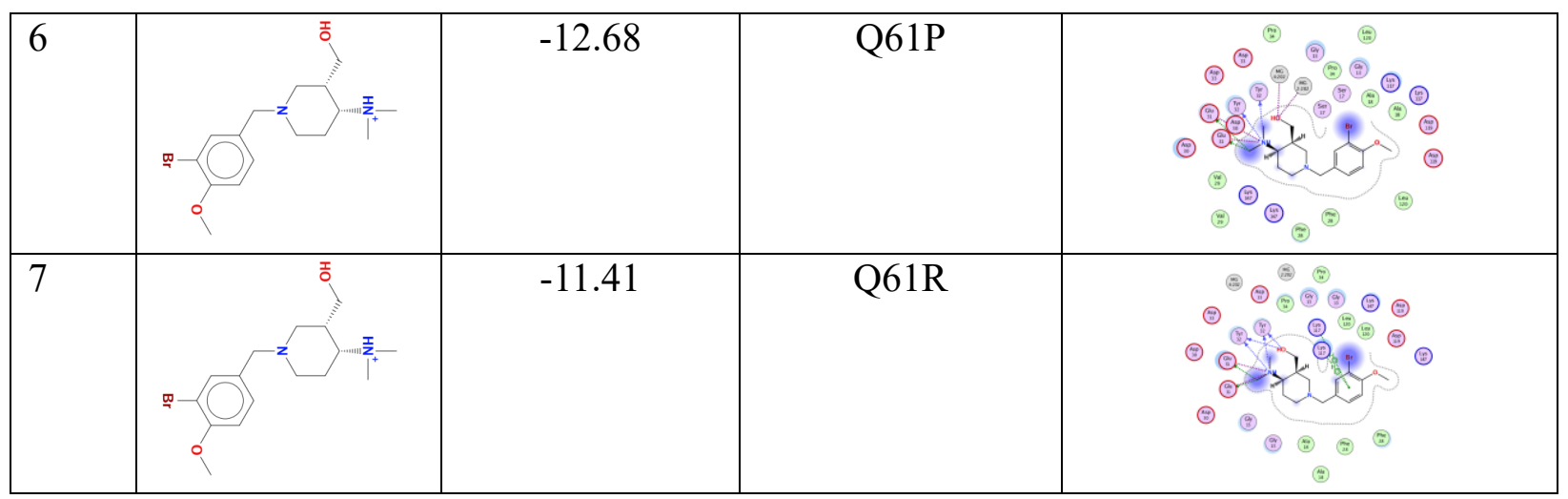

(ii) FH535-similar

\begin{tabular}{|c|c|c|c|c|}
\hline S.No. & Compound str. & Docking score & Mutation & Interaction \\
\hline 1 & 2 & -11.66 & Q61A & 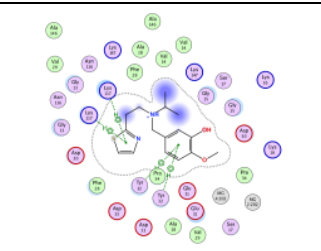 \\
\hline 2 & & -10.13 & Q61E & (2) \\
\hline 3 & < & -11.70 & Q61H & 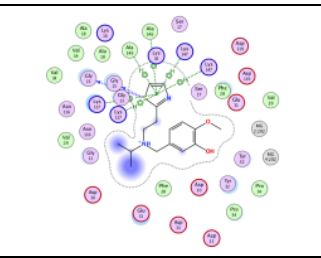 \\
\hline 4 & KC & -11.88 & Q61K & 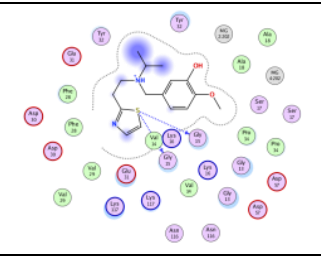 \\
\hline 5 & C & -14.17 & Q61L & 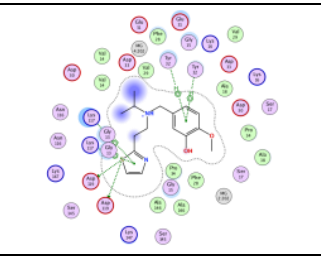 \\
\hline 6 & $\left\langle O_{n}^{2}\right.$ & -11.63 & Q61P & 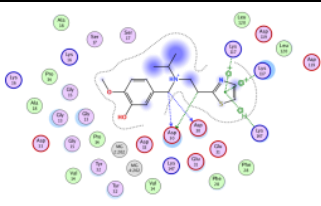 \\
\hline
\end{tabular}


Supporting Information

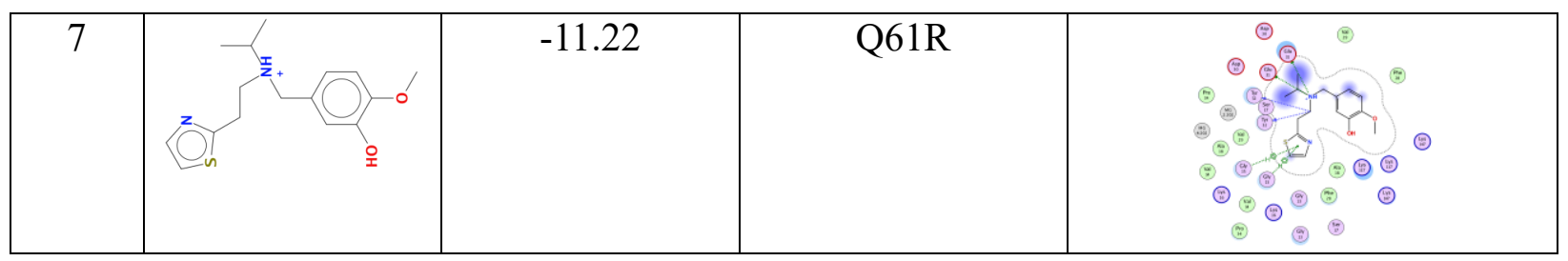

(iii) JQ1-similar

\begin{tabular}{|c|c|c|c|c|}
\hline S.No. & Compound str. & Docking score & Mutation & Interaction \\
\hline 1 & & -6.58 & Q61A & 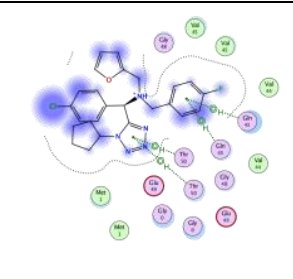 \\
\hline 2 & & -8.28 & Q61E & 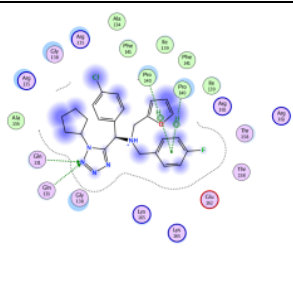 \\
\hline 3 & & -7.66 & Q61H & 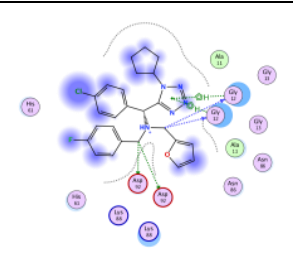 \\
\hline 4 & & -7.50 & Q61K & 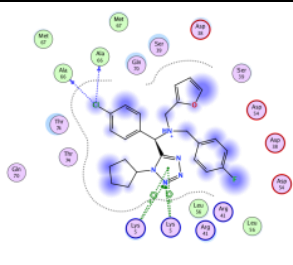 \\
\hline 5 & & -11.03 & Q61L & 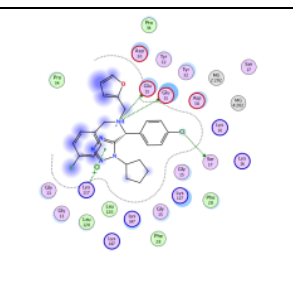 \\
\hline 6 & & -11.17 & Q61P & 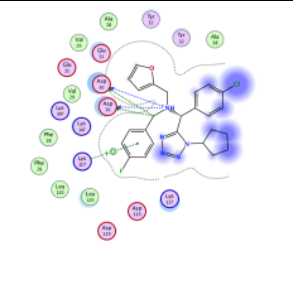 \\
\hline
\end{tabular}


Supporting Information

\begin{tabular}{|l|l|l|l|r|}
\hline 7 & -10.22 & Q61R & (3) \\
\hline
\end{tabular}

(iv) Olaparib-similar

\begin{tabular}{|c|c|c|c|c|}
\hline S.No. & Compound str. & Docking score & Mutation & Interaction \\
\hline 1 & & -12.28 & Q61A & 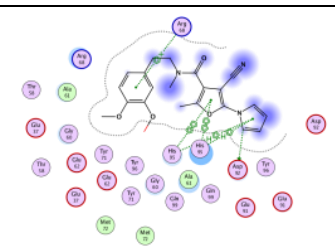 \\
\hline 2 & & -10.70 & Q61E & 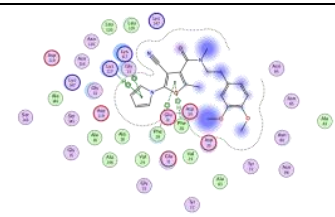 \\
\hline 3 & & -11.34 & Q61H & 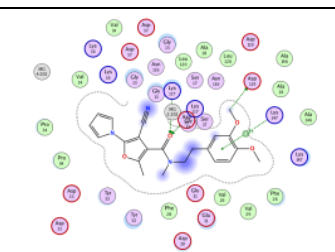 \\
\hline 4 & & -12.59 & Q61K & 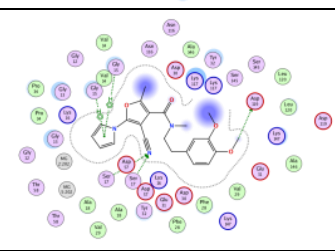 \\
\hline 5 & & -8.15 & Q61L & 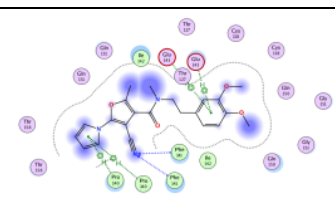 \\
\hline 6 & & -10.99 & Q61P & 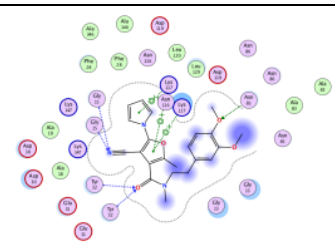 \\
\hline 7 & & -12.47 & Q61R & 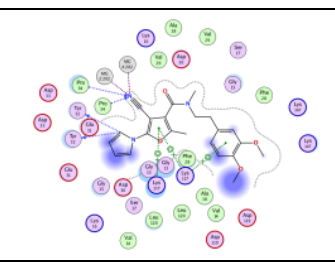 \\
\hline
\end{tabular}


Supporting Information

(v) PLX-4720-similar

\begin{tabular}{|c|c|c|c|c|}
\hline S.No. & Compound str. & Docking score & Mutation & Interaction \\
\hline 1 & & -12.80 & Q61A & 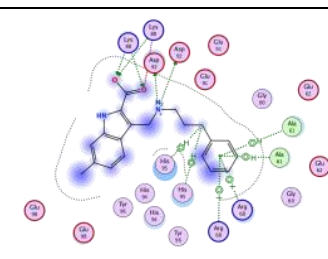 \\
\hline 2 & & -16.78 & Q61E & 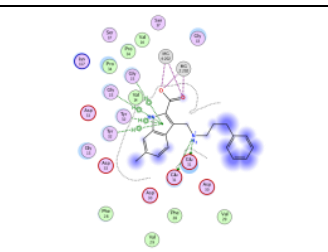 \\
\hline 3 & & -17.80 & Q61H & 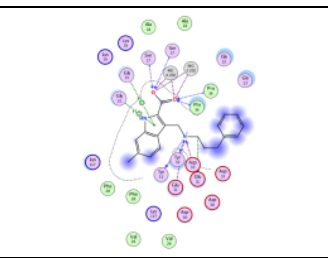 \\
\hline 4 & & -16.85 & Q61K & 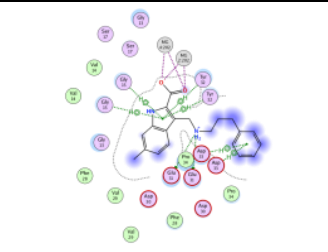 \\
\hline 5 & & -16.60 & Q61L & 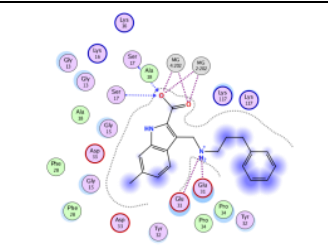 \\
\hline 6 & & -17.81 & Q61P & 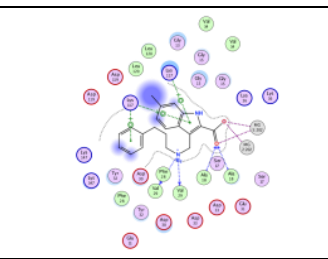 \\
\hline 7 & z & -18.11 & Q61R & 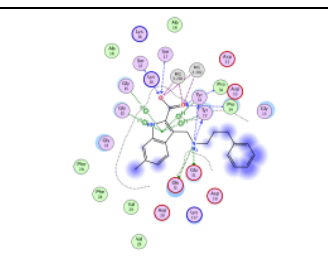 \\
\hline
\end{tabular}

(vi) PLX-4720-d7-similar

\begin{tabular}{|l|l|l|l|l}
\hline S.No. & Compound str. & Docking score & Mutation & Interaction \\
\hline
\end{tabular} 
Supporting Information

\begin{tabular}{|c|c|c|c|c|}
\hline 1 & $\int_{N-N}^{N} \prod_{0}^{H}$ & -9.35 & Q61A & 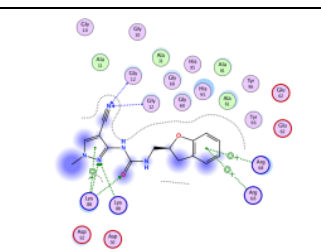 \\
\hline 2 & $\int_{N=N}^{N}$ & -11.16 & Q61E & 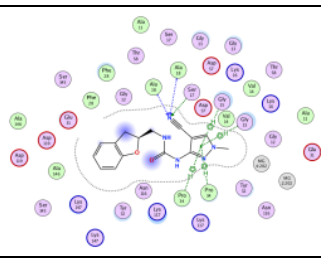 \\
\hline 3 & $\mathbb{N}$ & -11.15 & Q61H & 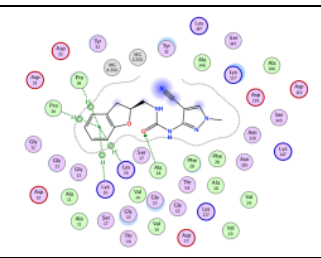 \\
\hline 4 & $N_{N}^{N-}$ & -9.67 & Q61K & 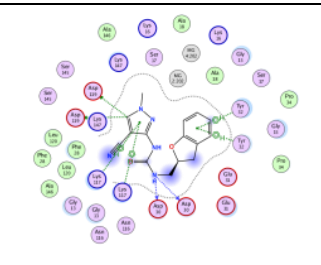 \\
\hline 5 & N- & -10.44 & Q61L & 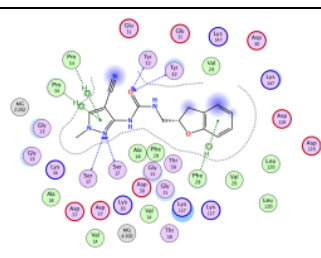 \\
\hline 6 & $\int_{N}-N$ & -11.30 & Q61P & 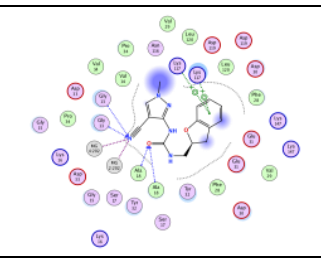 \\
\hline 7 & $\mathbb{N}$ & -11.32 & Q61R & 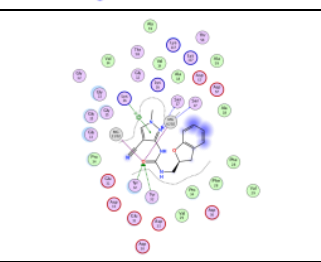 \\
\hline
\end{tabular}

(vii) Ruxolotinib-similar

\begin{tabular}{|l|l|l|l|l|}
\hline S.No. & Compound str. & Docking score & Mutation & Interaction \\
\hline
\end{tabular} 
Supporting Information

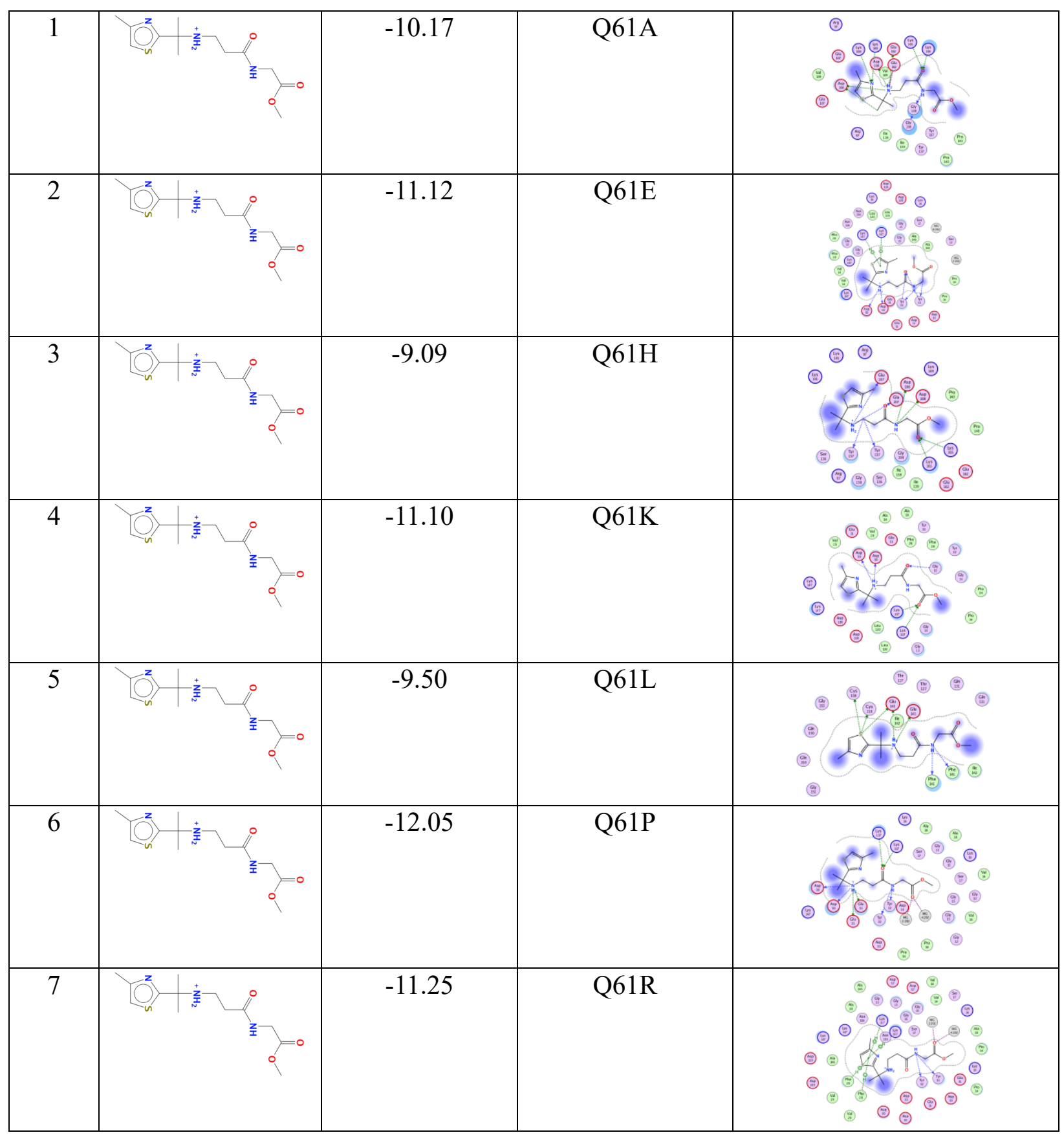

(viii) Tamoxifen-similar

\begin{tabular}{|c|c|c|c|c|}
\hline S.No. & Compound str. & Docking score & Mutation & Interaction \\
\hline 1 & & -11.16 & Q61A & \\
& & & &
\end{tabular}


Supporting Information

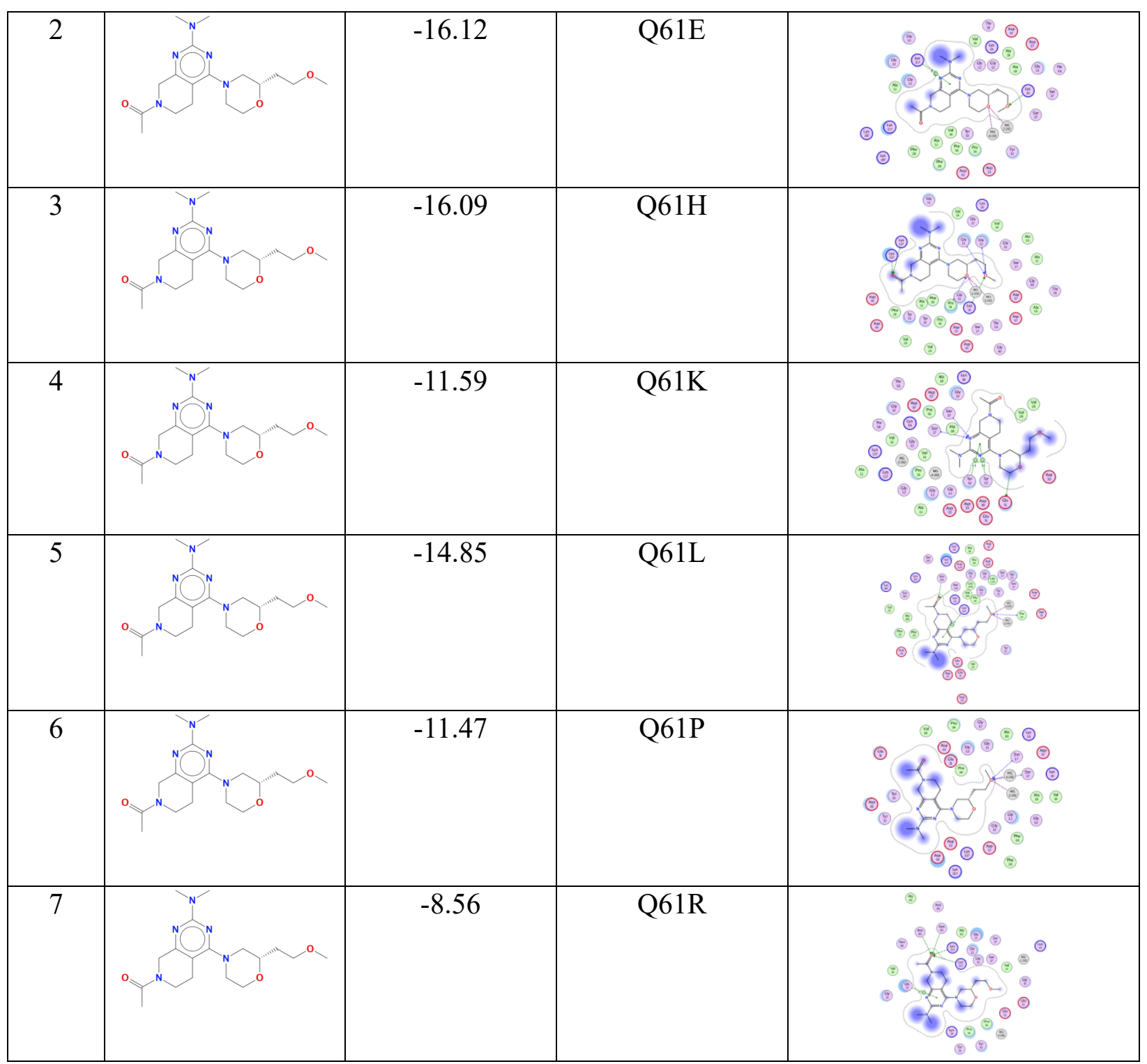

(ix) Vorinostat-similar

\begin{tabular}{|c|c|c|c|c|}
\hline S.No. & Compound str. & Docking score & Mutation & Interaction \\
\hline 1 & 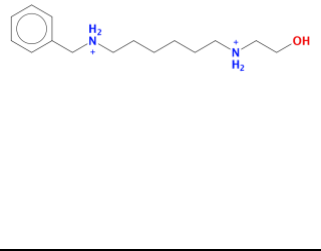 & -12.12 & Q61A & 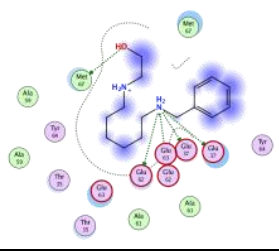 \\
\hline 2 & (1) $\mathrm{H}_{\mathrm{H}}^{\mathrm{H}_{2}} \mathrm{~N}_{\mathrm{H}_{2}}^{\mathrm{O}} \mathrm{OH}^{\mathrm{OH}}$ & -12.53 & Q61E & 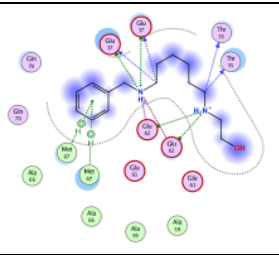 \\
\hline
\end{tabular}


Supporting Information

\begin{tabular}{|c|c|c|c|c|}
\hline 3 & (O) & -11.55 & Q61H & 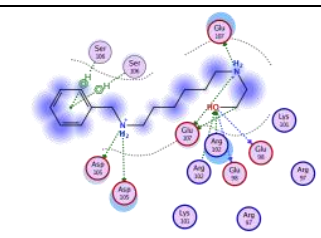 \\
\hline 4 & (1) $\mathrm{H}_{\mathrm{H}}^{\mathrm{H}_{2}} \mathrm{~N}_{\mathrm{H}_{2}}^{\mathrm{OH}}$ & -11.42 & Q61K & (i) \\
\hline 5 & 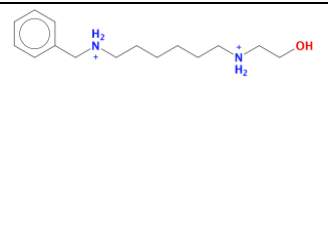 & -12.69 & Q61L & 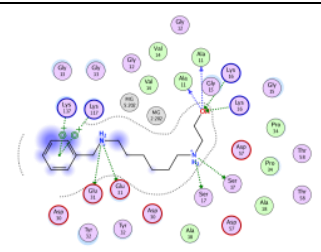 \\
\hline 6 & (1) & -11.14 & Q61P & 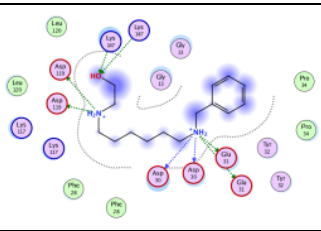 \\
\hline 7 & (1) & -11.77 & Q61R & 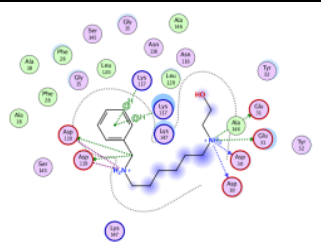 \\
\hline
\end{tabular}

(x) WZ-1-84-similar

\begin{tabular}{|c|c|c|c|c|}
\hline S.No. & Compound str. & Docking score & Mutation & Interaction \\
\hline 1 & & -11.07 & Q61A & 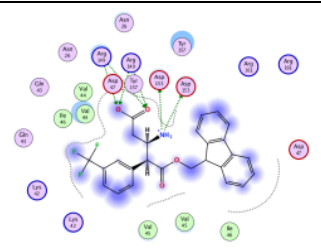 \\
\hline 2 & & -9.86 & Q61E & 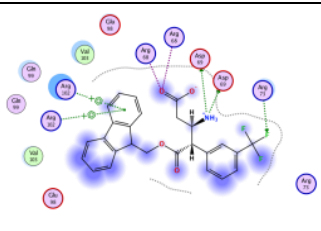 \\
\hline 3 & & -12.58 & Q61H & 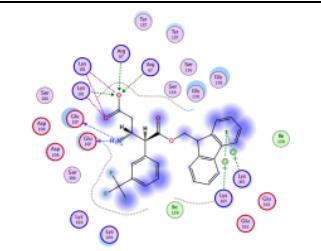 \\
\hline
\end{tabular}




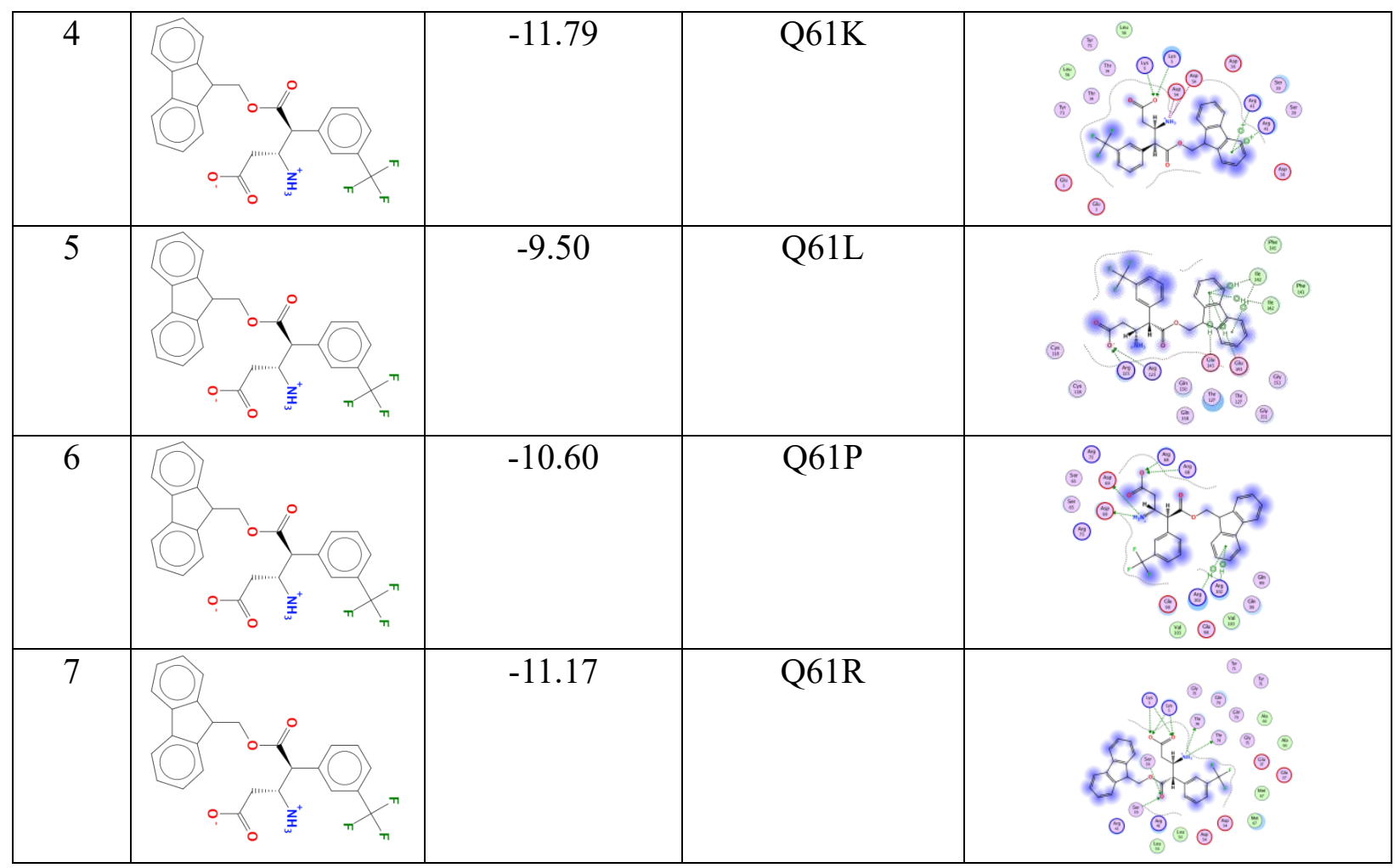

\section{REFERENCES}

1. Number, E. G. C., The relationship of K-ras mutations and EGFR gene copy number to outcome in patients treated with erlotinib on National Cancer Institute of Canada Clinical Trials Group trial study PA. 3. 2007.

2. Klapper, L. N.; Glathe, S.; Vaisman, N.; Hynes, N. E.; Andrews, G. C.; Sela, M.; Yarden, Y., The ErbB-2/HER2 oncoprotein of human carcinomas may function solely as a shared coreceptor for multiple stroma-derived growth factors. Proceedings of the National Academy of Sciences 1999, 96, 4995-5000.

3. Yarden, Y.; Schlessinger, J., Epidermal growth factor induces rapid, reversible aggregation of the purified epidermal growth factor receptor. Biochemistry 1987, 26, 1443-1451.

4. Zhang, H.; Berezov, A.; Wang, Q.; Zhang, G.; Drebin, J.; Murali, R.; Greene, M. I., ErbB receptors: from oncogenes to targeted cancer therapies. The Journal of clinical investigation 2007, 117, 2051-2058.

5. Tidyman, W. E.; Rauen, K. A., The RASopathies: developmental syndromes of Ras/MAPK pathway dysregulation. Current opinion in genetics \& development 2009, 19, 230236.

6. LoPiccolo, J.; Blumenthal, G. M.; Bernstein, W. B.; Dennis, P. A., Targeting the PI3K/Akt/mTOR pathway: effective combinations and clinical considerations. Drug Resistance Updates 2008, 11, 32-50.

7. $\quad$ Long, G. V.; Menzies, A. M.; Nagrial, A. M.; Haydu, L. E.; Hamilton, A. L.; Mann, G. J.; Hughes, T. M.; Thompson, J. F.; Scolyer, R. A.; Kefford, R. F., Prognostic and clinicopathologic 


\section{Supporting Information}

associations of oncogenic BRAF in metastatic melanoma. Journal of Clinical Oncology 2011, 29, 1239-1246.

8. Clark, D. A.; Coker, R., Transforming growth factor-beta (TGF-beta). The international journal of biochemistry \& cell biology 1998, 30, 293-298.

9. Liu, W.; Chang, B.-L.; Cramer, S.; Koty, P. P.; Li, T.; Sun, J.; Turner, A. R.; Von KapHerr, C.; Bobby, P.; Rao, J., Deletion of a small consensus region at 6q15, including the MAP3K7 gene, is significantly associated with high-grade prostate cancers. Clinical cancer research 2007, 13, 5028-5033.

10. Tournier, C.; Whitmarsh, A. J.; Cavanagh, J.; Barrett, T.; Davis, R. J., Mitogen-activated protein kinase kinase 7 is an activator of the c-Jun NH2-terminal kinase. Proceedings of the National Academy of Sciences 1997, 94, 7337-7342. 